\title{
Pollution State Modeling for Mexico City
}

\author{
Philip A. White ${ }^{1 *}$ | Alan E. Gelfand PhD ${ }^{1} \quad$ Eliane R. \\ Rodrigues PhD $^{2}$ | Guadalupe Tzintzun ${ }^{3}$
}

${ }^{1}$ Department of Statistical Science, Duke University, Durham, NC, United States of America

${ }^{2}$ Instituto de Matemáticas, Universidad Nacional Autónoma de México, Mexico

${ }^{3}$ Instituto Nacional de Ecología y Cambio Climático, Secretaría de Medio Ambiente y Recursos Naturales, Mexico

\section{Correspondence}

Philip White, Department of Statistical Science, Duke University, Durham, NC, 27708, United States of America

Email: philawhite@gmail.com

\section{Funding information}

The work of Eliane Rodrigues was partially funded by the project PAPIIT-IN102416 of the Dirección General de Apoyo al Personal Académico of the Universidad Nacional

Autónoma de México, Mexico

(DGAPA-UNAM).

Ground-level ozone and particulate matter pollutants are associated with a variety of health issues and increased mortality. For this reason, Mexican environmental agencies regulate pollutant levels. In addition, Mexico City defines pollution emergencies using thresholds that rely on regional maxima for ozone and particulate matter with diameter less than 10 micrometers $\left(\mathrm{PM}_{10}\right)$. To predict local pollution emergencies and to assess compliance to Mexican ambient air quality standards, we analyze hourly ozone and $\mathrm{PM}_{10}$ measurements from 24 stations across Mexico City from 2017 using a bivariate spatiotemporal model. Using this model, we predict future pollutant levels using current weather conditions and recent pollutant concentrations. Using hourly pollutant projections, we predict regional maxima needed to estimate the probability of future pollution emergencies. We discuss how predicted compliance to legislated pollution limits varies across regions within Mexico City in 2017. We find that predicted probability of pollution emergencies is limited to a few time periods. In contrast, we show that predicted exceedance of Mexican ambient air quality standards is a common, nearly daily occurrence.

\section{KEYWORDS}

Bayesian inference, environmental health, Mexico City, pollution monitoring, pollution regulation, spatiotemporal modeling

\footnotetext{
*Corresponding Author
} 


\section{1 | INTRODUCTION}

Long-term exposure to air pollution is strongly linked with respiratory and cardiovascular disease and leads to increased mortality as well as hospital admissions (see, e.g., Brunekreef and Holgate, 2002). Particulate matter (PM) is defined to be solid particles and liquid droplets in the air. PM comes from direct emissions (primary particles) and chemical reactions between other pollutants (secondary particles). Particulate matter, and in particular PM with diameter less than $10 \mu \mathrm{m}\left(\mathrm{PM}_{10}\right)$, is known to increase human mortality and morbidity (see, e.g., Brunekreef and Holgate, 2002; Pope III and Dockery, 2006; Loomis et al., 2013; Hoek et al., 2013). Because PM generally has a short lifetime, urban and other high emission areas generally have higher concentrations of $\mathrm{PM}_{10}$ than rural areas (see Clements et al., 2012, as an example).

Unlike PM, ground-level ozone $\left(\mathrm{O}_{3}\right)$ is not emitted directly but is instead formed by chemical reactions between of nitrogen oxides and volatile organic compounds, a reaction that requires heat and sunshine (see, e.g., Sillman, 1999). Ozone is often as high in rural areas as it is in urban areas (see, e.g., Angle and Sandhu, 1989; Sillman, 1999; Dueñas et al., 2004). Ozone is linked to a variety of negative health outcomes, including short-term respiratory events, long-term respiratory disease, increased mortality, and low birth weight (see, e.g., Lippmann, 1989; Salam et al., 2005; Bell et al., 2006; Weschler, 2006). Because of these adverse outcomes, regulatory agencies institute policies to monitor and limit pollution levels, especially $\mathrm{PM}_{10}$ and ozone. Urban areas are often monitored more closely to protect larger populations due to higher pollution levels found in urban environments (see, e.g., Heal and Hammonds, 2014). The detrimental health effects of air pollution in the Mexico City metropolitan area are well-studied (see Mage et al., 1996; Romieu et al., 1996; Hernández-Garduño et al., 1997; Loomis et al., 1999; Bravo-Alvarez and Torres-Jardón, 2002; Barraza-Villarreal et al., 2008; Riojas-Rodríguez et al., 2014). Thus, Mexican authorities have implemented a variety of regulations to control pollution levels in Mexico, and specifically in Mexico City.

In spite of several polices implemented by environmental authorities in Mexico and Mexico City over the past 30 years, the city and its metropolitan area still suffer with high levels of pollution (see, e.g., Bravo-Alvarez and TorresJardón, 2002; Zavala et al., 2009; Rodríguez et al., 2016; Davis, 2017; Instituto Nacional de Ecología y Cambio Climático (INECC), 2017; Gouveia et al., 2018). Some of the most recent measures implemented are new thresholds limiting ozone and $\mathrm{PM}_{10}$ concentrations nation-wide which decreased allowable pollution levels relative to previous thresholds (Diario Oficial de la Federación, 2014a,b). Thresholds are updated every five years based on current research on the effect of pollutants on human health. In these new standards, the ozone thresholds were reduced to 95 parts per billion (ppb) or, equivalently, 0.095 parts per million ( $\mathrm{ppm}$ ) for hourly ozone and 70 ppb for eight-hour average ozone (Diario Oficial de la Federación, 2014b). Additionally, the allowable 24-hour average PM 10 concentration threshold was lowered to 75 micrograms per cubic meter $\left(\mu \mathrm{g} / \mathrm{m}^{3}\right.$ ) (Diario Oficial de la Federación, 2014a). These thresholds are not used to reduce pollution levels but are instead established to ensure human health protection and to evaluate air quality.

By comparison, the United States Environmental Protection Agency (EPA) limits 24-hour average PM 10 concentration to not exceed $150 \mu \mathrm{g} / \mathrm{m}^{3}$ and 8-hour average ozone concentration to not exceed $70 \mathrm{ppb}$. (101st United States Congress, 1990). The European Union restricts 24-hour average $\mathrm{PM}_{10}$ concentration to not exceed $50 \mu \mathrm{g} / \mathrm{m}^{3}$ and 8-hour average ozone concentration to not exceed 120 ppb (European Environment Agency, 2016). Thus, Mexican ambient air quality standards (which we denote MAAQS) are progressive when compared to American and European standards. Mexico City's pollution emergencies, however, are not related to the Mexican national standards and instead use more permissive thresholds.

Mexico City's thresholds, established by the Atmospheric Environmental Contingency Program in Mexico City, are used to indicate times when pollutant concentrations are high enough to cause significant damage to human health (Administración Pública de la Ciudad de México, 2016). Thus, the goals of Mexico City's Atmospheric Environmental 
Contingency Program differ from those specified for Mexico's ambient air quality standards. When emergency phases (or events) are activated, the aim is to control emission levels to decrease air pollution and its harmful effects to the population. It is worth mentioning that thresholds have decreased significantly. For instance, the thresholds for declaring the equivalent emergencies 1995-2000 were 1.5-2 times the current limits, depending on the type of emergency declared (Departamento del Distrito Federal et al., 1996).

Mexico City and its metropolitan area are split into five regions: northeast (NE), northwest (NW), central (CE), southeast (SE), and southwest (SW). Within these five regions, there are 24 monitoring stations that record both hourly ozone and $\mathrm{PM}_{10}$ levels during the year 2017. To control the health risks associated with high ozone and $\mathrm{PM}_{10}$, environmental alerts are declared if either hourly ozone or 24-hour average $\mathrm{PM}_{10}$ levels exceed certain pollutant-specific thresholds which rely on regulatory suggestions that differ from those presented in Diario Oficial de la Federación (2014a,b). Depending on the levels of the pollutant, either a phase I or a phase II alert is declared. Phase I is declared when hourly ozone exceeds $L_{1}^{O}=0.154 \mathrm{ppm}\left(154 \mathrm{ppb}\right.$ ) or 24-hour average $\mathrm{PM}_{10}$ exceeds $L_{1}^{P M}=214 \mu \mathrm{g} / \mathrm{m}^{3}$. During a phase l emergency, people are encouraged to limit outdoor time, exercise, smoking, and consumption of gas. Additionally, several transportation protocols are instituted to reduce vehicular emissions. Similarly, phase II is declared when hourly ozone exceeds $L_{2}^{O}=0.204 \mathrm{ppm}$ (204 ppb) or 24-hour average $\mathrm{PM}_{10}$ exceeds $L_{2}^{P M}=354 \mu \mathrm{g} / \mathrm{m}^{3}$. Phase II institutes stricter protocols than phase I, including restricting circulation of official vehicles and strictly limiting civilian and commercial emissions. See Administración Pública de la Ciudad de México (2016) for details regarding Mexico City's pollution emergency phases.

Compared to MAAQS, Mexico City's Atmospheric Environmental Contingency Program thresholds are more tolerant of high pollution levels. The phase I thresholds for ozone are 1.6 times the Mexican legal limits, while the phase I thresholds for $\mathrm{PM}_{10}$ are almost three times MAAQS. The phase II thresholds are roughly two and five times MAAQS for ozone and $\mathrm{PM}_{10}$, respectively. The protocols for phase I or phase II are the same regardless of the pollutant that triggered the alert. If ozone thresholds are exceeded in any region, i.e., the maximum over any station within the region, then emergency phases are declared city-wide (i.e., in all regions), where the phase is determined by which threshold $\left(L_{1}^{O}\right.$ or $\left.L_{2}^{O}\right)$ was exceeded.

On the other hand, $\mathrm{PM}_{10}$ exceedances could trigger regional or city-wide phase alerts, depending on which stations exceed the allowable limits. More explicitly, if the maximum 24-hour average over stations within the same region exceeds a $\mathrm{PM}_{10}$ threshold, then the environmental alert is declared only in that region. However, if the maxima for two or more regions exceed a given $\mathrm{PM}_{10}$ threshold, then the environmental alert is declared over the entire metropolitan area (i.e. in all five regions). This description is summarized in Table 1.

\begin{tabular}{|l|l|l|}
\hline Phase & Region-wide Alert & City-wide Alert \\
\hline None & $\begin{array}{l}\bullet \mathrm{PM}_{10}<L_{1}^{P M} \text { and } O_{3}<L_{1}^{O} \text { for all regions } \\
\bullet \text { No higher-order alerts supersede }\end{array}$ & $\bullet P M_{10}<L_{1}^{P M}$ and $O_{3}<L_{1}^{O}$ for all regions \\
\hline I & $\bullet P M_{10} \geq L_{1}^{P M}$ within the region & $\bullet O_{3} \geq L_{1}^{O}$ for any region \\
& $\bullet$ And no higher-order alerts supersede & $\bullet$ Or $\mathrm{PM}_{10} \geq L_{1}^{P M}$ for two or more regions \\
\hline II & $\bullet \mathrm{PM}_{10} \geq L_{2}^{P M}$ within the region & $\bullet$ And no higher-order alerts supersede \\
\hline & & $\bullet O_{3} \geq L_{2}^{O}$ for any region \\
\hline
\end{tabular}

TAB LE 1 Description of Mexico City emergency phase alerts. Note that ozone thresholds are for hourly ozone, while $\mathrm{PM}_{10}$ limits are for 24-hour running average $\mathrm{PM}_{10}$. 
Pollution emergency phases are only suspended when pollution levels for every station drop below phase I thresholds (i.e. the conditions for no phase alerts are met). For practical reasons, evaluation of the emergency phases is carried out three times daily at 10 AM, 3 PM, and 8 PM (Administración Pública de la Ciudad de México, 2016). Ultimately, however, phase activation and suspension are dependent on meteorological forecasts in addition to observed pollution levels. Because the additional meteorological criteria are not explicitly outlined, we do not attempt to predict actual phase occurrence but instead quantify the risk of a phase occurrence.

The contribution here is to understand and predict how often Mexico City was at risk of a pollution emergency in terms of (1) the Atmospheric Environmental Contingency Program in Mexico City and (2) current Mexican ambient air quality standards. For both, we assess how the risk of dangerous pollution varies over city regions and over time. As described above, for Mexico City's Atmospheric Environmental Contingency Program, alerts are triggered when one or more stations exceeds thresholds. Thus, emergency phases depend entirely upon pollutant maxima within each region. Furthermore, environmental alerts are often summarized over coarser temporal scales, like days, rather than the measurement level (hours) or the three hours of evaluation (10 AM, 3 PM, and 8 PM). So, daily emergency phases depend on pollutant maxima over hours of evaluation and stations within each region. Regarding MAAQS, we can do inference at each of three natural spatial scales: station-level, region-level, or city-level. Again, we may be interested in exceedances occurring on a daily scale rather than hourly. Therefore, we again need maxima over time (and potentially space depending on the spatial scale selected).

In summary, the foregoing tasks addressed here, the analyses of emergency contingency plan and Mexican ambient air quality standards, rely on the same pollution level data. Therefore, we develop, using model choice over a selection of models, a single hierarchical bivariate spatiotemporal model for hourly ozone and $\mathrm{PM}_{10}$ levels. Predictions from our model serve two practical purposes: First, our predictions allow us to carry out probabilistic inference about pollution emergency states or national compliance issues. Second, if implemented in practice, our model could warn of potential pollution emergencies or compliance problems, allowing regional and city-wide adjustments and responses to be made earlier. From this model, all prediction and inference regarding emergency phases and legislation-based exceedances becomes a post-model fitting exercise, as we demonstrate.

By now there is a rich literature on modeling both $\mathrm{O}_{3}$ and $\mathrm{PM}$ at both coarse $(10 \mu \mathrm{m})$ and fine $(2.5 \mu \mathrm{m})$ scale. Here, we highlight some examples relevant to our analysis. Sahu et al. (2007) use a hierarchical space-time model to model square-root ozone with the goal of assessing long term trends in ozone in Ohio. Cocchi et al. (2007) adopt a hierarchical model for log-PM 10 concentrations to characterize the effect of meteorological conditions on the PM 10 process and to estimate $\mathrm{PM}_{10}$ at unmonitored locations. Berrocal et al. (2010) model square-root ozone using data of two types, output from numerical models and data collected from monitoring networks, that are misaligned on spatial scales using spatially-varying regression coefficients (Gelfand et al., 2003). Huang et al. (2018) model log-PM 10 and log-nitrogen dioxide $\left(\mathrm{NO}_{2}\right)$ jointly and assessed the effect of these pollutants on health outcomes in Scotland. Similarly, we adopt a hierarchical space-time model with site-specific regression and auto-regressive coefficients for square-root ozone and log-PM 10 concentrations in Mexico City, Mexico.

In this paper, we start by presenting and discussing the Mexico City pollution dataset in Section 2, highlighting data characteristics that inform modeling decisions. In Section 3, we discuss modeling decisions, model fitting, inference, and selection. We present and discuss results in the context of both Mexico City's Atmospheric Environmental Contingency Program and MAAQS in Section 4. In this section, we first present a comprehensive analysis of Mexico City's phase alert system, predicting pollution levels and associated phase levels at 10 AM, 3 PM, and 8 PM to mirror the actual phase activation and suspension procedure. Then, we carry out inference on MAAQS exceedances and compare these results to emergency phase predictions to demonstrate differences between these standards. We provide concluding discussion regarding our results and statistical modeling in Section 5. 


\section{2 | MEXICO CITY POLLUTION DATASET}

In this dataset, we have hourly ozone and $\mathrm{PM}_{10}$ measurements at $N_{s}=24$ stations across Mexico City, Mexico for the duration of 2017. Ozone and $\mathrm{PM}_{10}$ measurements are obtained minute by minute at each station, and the hourly measurement reported is an average of the 60 minute-by-minute measurements. Let $Y_{i t}^{O}, Y_{i t}^{P M}$ denote ozone and $P M 10$ levels, respectively, at station $i$ and time $t$ with units of hours. Consequently, we observe measurements over $N_{t}=8760$ times at each station, giving $N=210240$ pairs of ozone and $\mathrm{PM}_{10}$ concentration across the 24 stations, across the entire year. ${ }^{1}$ Relative humidity $(\mathrm{RH})$ and temperature (TMP) are measured over the same space-time grid as ozone and $\mathrm{PM}_{10}$ and are used as explanatory variables for both ozone and $\mathrm{PM}_{10}$.

As mentioned above, Mexico City is partitioned into five regions which are employed for defining environmental alert phases (See Section 1). Abbreviated station names, corresponding regions, and annual summaries of pollution levels are given in Table 2. Station locations are plotted in Figure 1 using the R package GGMAP (Kahle and Wickham, 2013). Besides being on the main wind path (from NE to SW) and therefore receiving many ozone precursors from the NE region, the CE region is heavily-trafficked by automobile. The SW region, located and the end of the NE-SW wind corridor, receives ozone produced along this wind path, and this ozone stays trapped in the SW region due to mountains on its southwest boundary.

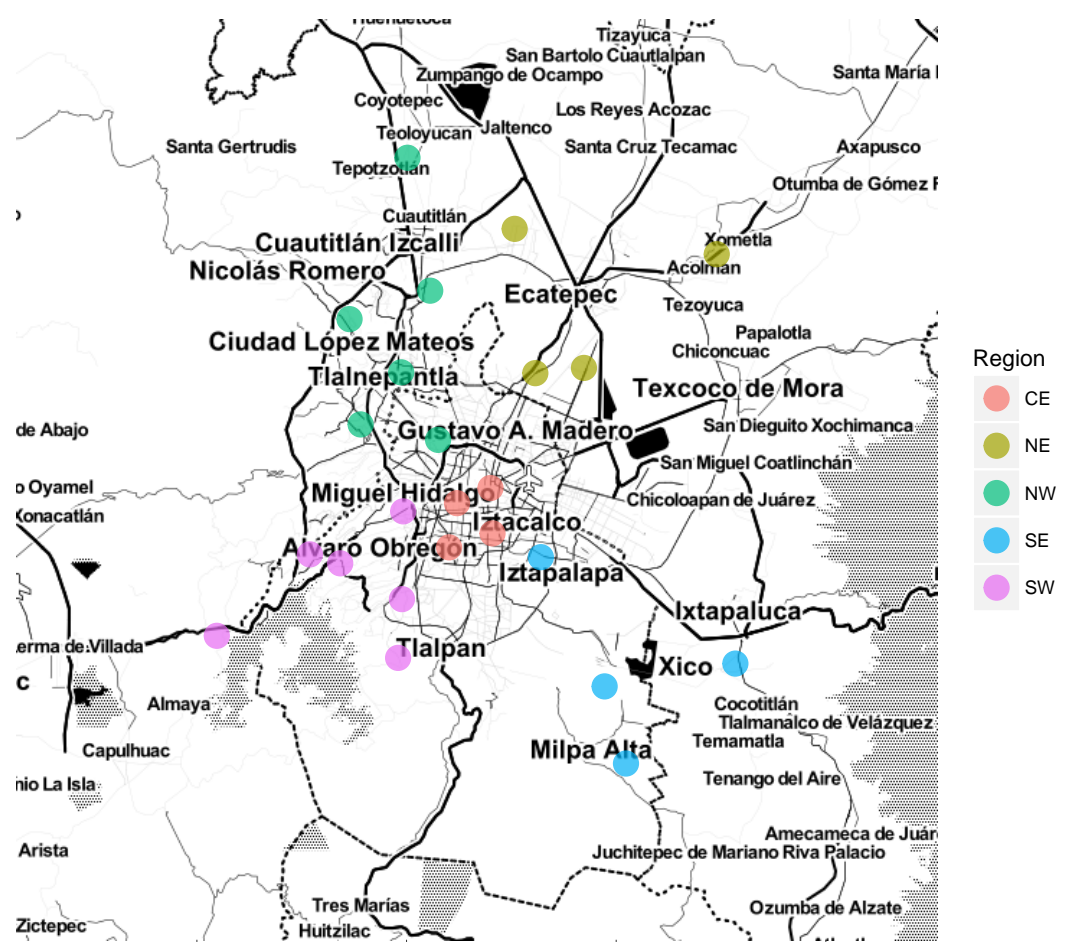

FIGURE 1 Station locations with regional labels.

\footnotetext{
${ }^{1}$ Missing hourly measurements were imputed using the corresponding measurements at the nearest station within the same region. If no stations in that region recorded a measurement at that time, then the nearest station in a different region provided the missing value. This was done prior to our receiving the data for analysis.
} 


\begin{tabular}{rclcc}
\hline Region & Stations & Station Names & $\begin{array}{c}\text { Annual } \\
\text { Average Ozone }\end{array}$ & $\begin{array}{c}\text { Annual } \\
\text { Average PM } 10\end{array}$ \\
\hline Northeast & 4 & ACO, SAG, VIF, XAL & $28 \mathrm{ppb}$ & $59 \mu \mathrm{g} / \mathrm{m}^{3}$ \\
\hline Northwest & 6 & ATI, CAM, CUT, & $27 \mathrm{ppb}$ & $49 \mu \mathrm{g} / \mathrm{m}^{3}$ \\
\hline Central & 4 & BJU, HGM, IZT, MER & $29 \mathrm{ppb}$ & $44 \mu \mathrm{g} / \mathrm{m}^{3}$ \\
\hline Southeast & 4 & CHO, MPA,TAH, UIZ & $36 \mathrm{ppb}$ & $45 \mu \mathrm{g} / \mathrm{m}^{3}$ \\
\hline Southwest & 6 & AJM, CUA, INN & $34 \mathrm{ppb}$ & $33 \mu \mathrm{g} / \mathrm{m}^{3}$ \\
& & MGH, PED, SFE & & \\
\hline
\end{tabular}

TAB LE 2 Station names and regions. Average ozone and $\mathrm{PM}_{10}$ across regions are given.

Note that the number of stations in each region differs. Moreover, pollution levels appear to vary over the regions. The northeast region has the highest average $\mathrm{PM}_{10}$, while the southeast and southwest regions have the highest average ozone. Hence, the regional maxima, used for phase alerts, are expected to have very different hourly distributions. Note that, with the maxima being taken over a small number of stations in each region, there is no reason to attempt to employ extreme value theory here. We model at the station-level rather than at the regional level so that the regional maximum distributions are induced by the station-level modeling. In this regard, because ozone and $\mathrm{PM}_{10}$ are strictly non-negative, we consider modeling the station data using either transformations to $\mathbb{R}$ or using strictly positive data models.

Region-specific box-plots for ozone and $\mathrm{PM}_{10}$ are plotted in Figures 2a and 2b. For ozone, the SE region has the highest mean, but the CE and SW regions have the most extreme values. Note that the NE region has the highest average $\mathrm{PM}_{10}$, as well as the most extreme values. This is because the NE region houses a large industrial section that generates many direct pollutants, including particulate matter. To explore the relationships between covariates ( $\mathrm{RH}$ and TMP), outcomes (ozone and $\mathrm{PM}_{10}$ ), and covariates and outcomes, we compute the station-specific Spearman's $\rho$ for all covariate-covariate, covariate-outcome, and outcome-outcome relationships. As a rank correlation, Spearman's $\rho$ avoids concern regarding transformations and outlying values. We plot these site-specific correlation coefficients in Figure 2c.

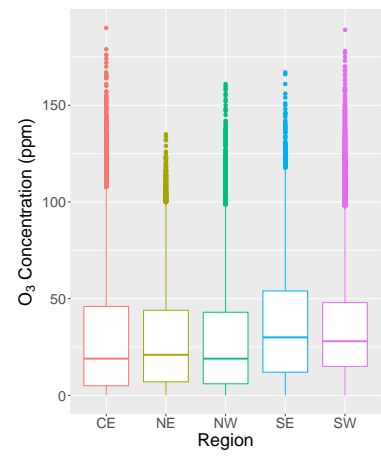

(a) Region $\mathrm{O}_{3}$ boxplot

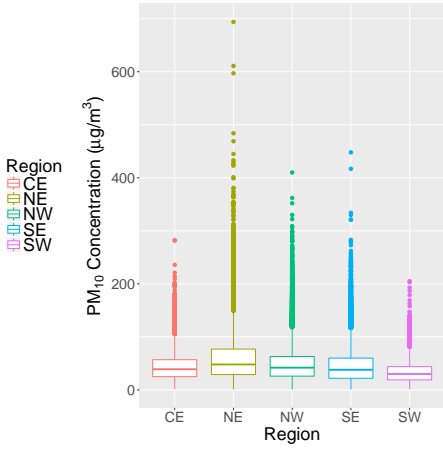

(b) Regional $\mathrm{PM}_{10}$ boxplot

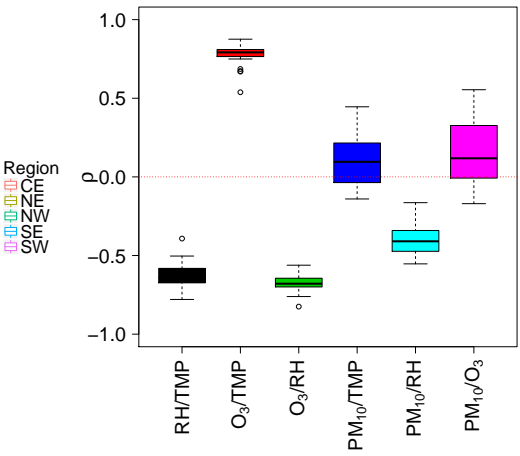

(c) Correlation boxplots

FI GURE 2 Region-specific boxplots for (Left) ozone and (Center) PM 10 . (Right) Site-specific Spearman's $\rho$ for (from left to right) relative humidity and temperature, ozone and temperature, ozone and relative humidity, $\mathrm{PM}_{10}$ and temperature, $\mathrm{PM}_{10}$ and relative humidity, and $\mathrm{PM}_{10}$ and ozone. 
The relationships between ozone and covariates (RH and TMP) are strong for all sites, while the relationships between $\mathrm{PM}_{10}$ and covariates (RH and TMP) vary much more across sites. However, there appears to be a strong negative correlation between $\mathrm{RH}$ and $\mathrm{PM}_{10}$ for all locations. On the other hand, Spearman's $\rho$ varies greatly across sites for $\mathrm{TMP}$ and $\mathrm{PM}_{10}$. Similarly, there appear to be important relationships between ozone and $\mathrm{PM}_{10}$ depending on the site, motivating the use of a joint model for ozone and $\mathrm{PM}_{10}$. The variability of the outcome, covariate, and outcome-covariate relationships across stations motivates a hierarchical model for covariate effects.

We find strong daily and weekly patterns for both pollutants. Using residual analysis for a model with site-specific effects for meteorological covariates, we still observe strong seasonal patterns for both day and week. Additionally, our preliminary analyses reveal strong correlation between the variance of residuals and the mean for both pollutants. This correlation could be addressed through modeling in a variety of ways. First, and most simply, one could use a variance stabilizing transformation (VST) to address the correlation between the mean and variance (e.g. log, square-root, Box-Cox) as was done by, for example, Sahu et al. (2007); Cocchi et al. (2007); Berrocal et al. (2010); Huang et al. (2018). Alternatively, we could use heteroscedastic models that specify variance directly as a function of hour or month. We consider both modeling approaches (transformations and heteroscedasticity) in Section 3.3. This exploratory analysis is provided in the online supplement.

\section{3 | METHODS AND MODELS}

Given the exploratory analysis, a time-series analysis is certainly warranted. Because the data are collected hourly, because the exposure standards are at the scale of hours (or functions of hours), and because we can identify useful discrete lags which are difficult to capture with covariance specifications, we elect to work with discrete time rather than continuous time. Additionally, our exploratory analysis suggests that a model using either a VST or time-varying variance may describe the data more accurately than models using non-transformed data or homoscedastic models. As a result, we envision the model for these data to be

$$
\begin{gathered}
Y_{i t}^{O}=\mathbf{x}_{i(t-1)}^{T} \boldsymbol{\beta}_{1 i}+\mathbf{L}_{i t}^{O^{T}} \gamma_{1 i}+\psi_{1 i}+\epsilon_{1 i t} \\
Y_{i t}^{P M}=\mathbf{x}_{i(t-1)}^{T} \boldsymbol{\beta}_{2 i}+\mathbf{L}_{i t}^{P M^{T}} \gamma_{2 i}+\psi_{2 i}+\epsilon_{2 i t},
\end{gathered}
$$

where $Y_{i t}^{O}$ is ozone concentration (or square-root ozone) and $Y_{i t}^{P M}$ is $\mathrm{PM}_{10}$ concentration (or log-PM10) at site $i$ and hour $t$. Here, $\mathbf{x}_{i(t-1)}$ includes an intercept, temperature, and relative humidity at site $i$ and time $t-1$. We use covariates from the previous hour because one of the primary purposes of this model is one-hour-ahead predictions for pollutants and corresponding phase alerts and exceedance probabilities and $\mathbf{x}_{i t}$ will not be available for such prediction.

The parameters $\boldsymbol{\beta}=\left(\boldsymbol{\beta}_{11}, \ldots, \boldsymbol{\beta}_{1 N_{s}}, \boldsymbol{\beta}_{21}, \ldots, \boldsymbol{\beta}_{2 N_{s}}\right)$ are station-specific regression coefficients for both $\mathrm{PM}_{10}$ and ozone. Because we imagine that the effect of humidity on pollutant concentrations is similar from region to region, we model regression coefficients exchangeably and hierarchically, centering effects on respective common means (see Gelman et al., 2014, for introductory thoughts on such hierarchical modeling). We define $\mathbf{L}_{i t}^{O}$ and $\mathbf{L}_{i t}^{P M}$ to be generic vectors of the lagged observations for ozone and $\mathrm{PM}_{10}$, respectively with $\gamma=\left(\gamma_{11}, \ldots, \gamma_{1 N_{s}}, \gamma_{21}, \ldots, \gamma_{2 N_{s}}\right)$ as corresponding site-specific autoregressive coefficients. Lags for observations in early January $2017\left(\mathrm{~L}_{i t}^{O}\right.$ and $\mathrm{L}_{i t}^{P M}$ ) may depend upon observations from December 2016. The choice of components of $\mathbf{L}_{i t}^{O}$ and $\mathbf{L}_{i t}^{P M}$ becomes the model choice issue which we take up in Section 3.3. As with $\boldsymbol{\beta}$, we model $\boldsymbol{\gamma}$ hierarchically. Then, we have pure error terms, $\epsilon_{1 i t} \stackrel{i i d}{\sim} N\left(0, \sigma_{1}^{2}\right)$ and $\epsilon_{2 i t} \stackrel{i i d}{\sim} N\left(0, \sigma_{2}^{2}\right)$, or $\epsilon_{1 i t} \stackrel{i n d}{\sim} N\left(0, \sigma_{1 t}^{2}\right)$ and $\epsilon_{2 i t} \stackrel{i n d}{\sim} N\left(0, \sigma_{2 t}^{2}\right)$ for the heteroscedastic formulation.

Finally, to bring in spatial structure across the sites, jointly, $\psi_{1 i}, \psi_{2 i}$ follow a bivariate conditionally autoregressive 
(CAR) model using coregionalization of two independent CAR models $V_{1 i}$ and $V_{2 i}$ (see Rue and Held, 2005; Banerjee et al., 2014). Coregionalization allows flexible, multivariate modeling (see, e.g., Matheron, 1982; Grzebyk and Wackernagel, 1994; Wackernagel, 1994; Banerjee et al., 2014). Explicitly,

$$
\begin{aligned}
\left(\begin{array}{l}
\psi_{1 i} \\
\psi_{2 i}
\end{array}\right) & =\mathbf{A}_{\psi}\left(V_{1 i}, V_{2 i}\right)^{T} \\
\mathbf{A}_{\psi} & =\left(\begin{array}{ll}
a_{11}^{(\psi)} & 0 \\
a_{12}^{(\psi)} & a_{22}^{(\psi)}
\end{array}\right)
\end{aligned}
$$

where $\mathbf{V}_{1}=\left(V_{11}, V_{12}, \ldots, V_{1 N_{s}}\right)^{T}$ and $\mathbf{V}_{2}=\left(V_{21}, V_{22}, \ldots, V_{2 N_{s}}\right)^{T}$. Equivalently, we can view $a_{11}^{(\psi)}$ and $a_{22}^{(\psi)}$ as scale parameters for the $\mathbf{V}_{1}$ and $\mathbf{V}_{2}$, a fact we use in model fitting (See Appendix $A$ ). Because there are not natural borders or edges shared between stations, it is natural that $\mathbf{V}_{1}$ and $\mathbf{V}_{2}$ would use an inverse distance-dependent proximity matrix which we denote $\mathbf{W}$. We assume the same distant-dependent CAR structure for both $\mathbf{V}_{1}$ and $\mathbf{V}_{2}$, where weights are proportional to $\exp (-a d)$ with $d$ denoting the distance between locations and a being the inverse of the maximum distance between stations. For common proximity matrix $\mathbf{W}$, if we let $D_{W}$ be diagonal with $\left(D_{W}\right)_{i i}=w_{i+}$, where $w_{i+}=\sum_{i} \mathbf{W}_{i j}$, then, the (unscaled) precision matrix of $\mathbf{V}_{1}$ and $\mathbf{V}_{2}$ is $Q=D_{W}-W$.

\section{1 | Priors, Model Fitting, and Prediction}

We model regression and autoregressive coefficients $\boldsymbol{\beta}_{1 i}, \boldsymbol{\beta}_{2 i}, \boldsymbol{\gamma}_{1 i}$, and $\boldsymbol{\gamma}_{2 i}$ hierarchically,

$$
\begin{array}{llll}
\boldsymbol{\beta}_{1 i} \sim N\left(\boldsymbol{\beta}_{01}, \Sigma_{\beta_{1}}\right), & \gamma_{1 i} \sim N\left(\gamma_{01}, \Sigma_{\gamma_{1}}\right), & & \Sigma_{\beta_{1}} \sim I W\left(10^{3} \mathrm{I}, p+1\right), \\
\boldsymbol{\beta}_{2 i} \sim N\left(\boldsymbol{\beta}_{02}, \Sigma_{\beta_{2}}\right), & \gamma_{2 i} \sim N\left(\gamma_{02}, \Sigma_{\gamma_{2}}\right), & & \Sigma_{\beta_{2}} \sim I W\left(10^{3} \mathrm{I}, p+1\right), \\
\boldsymbol{\beta}_{01} \sim N\left(\mathbf{0}, 10^{3} \mathrm{I}\right), & \gamma_{01} \sim N\left(0,10^{3} \mathrm{I}\right), & \Sigma_{\gamma_{1}} \sim I W\left(10^{3} \mathrm{I}, n_{1 /}+1\right), \\
\boldsymbol{\beta}_{02} \sim N\left(\mathbf{0}, 10^{3} \mathrm{I}\right), & \gamma_{02} \sim N\left(\mathbf{0}, 10^{3} \mathrm{I}\right), & \Sigma_{\gamma_{2}} \sim I W\left(10^{3} \mathrm{I}, n_{2 I}+1\right),
\end{array}
$$

where $p=3$ is the number of regressors including the intercept, $n_{1 /}$ is the number of lags for ozone, and $n_{2 /}$ is the number of lags for $\mathrm{PM}_{10}$. By this, we assume that station-specific regression and autoregression coefficients are exchangeable. For the variance terms in the likelihood and the CAR prior, we assume that

$$
\begin{array}{lll}
\sigma_{1}^{2} \sim I G(1,1), & a_{11}^{(\psi)^{2}} \sim I G(1,1), \\
\sigma_{2}^{2} \sim I G(1,1), & a_{22}^{(\psi)^{2}} \sim I G(1,1) .
\end{array}
$$

Lastly, we assume $a_{12}^{(\psi)^{2}} \sim N\left(0,10^{3}\right)$. Model fitting details via a Gibbs sampler are given in Appendices $A$ and $B$. This model could be fit sequentially, but this would require us to update model parameters 8760 times for each step an MCMC sampler, once for each hour in 2017.

Prediction can be done in two ways, each with a different purpose: one predicts at unobserved values within the time range of the data (missing data) or one can predict future observations (forecasting). The former is viewed as retrospective prediction, filling in missing data over sites and times. The latter is viewed as prospective, predicting the next hour given the data up to the current hour. We are interested in MAAQS exceedances for specific months on an hourly scale. In this case, we only train model parameters on data observed prior to our predictions. Instead of a fully sequential model fitting where the model is updated hourly, we fit the model up to the last hour of the previous 
month. This model is then used to predict for pollutant levels for the upcoming month, making all prediction in this setting prospective. When carrying out inference for all days simultaneously, we fit the model to all the data once. For pollution and phase predictions, we limit our prediction to 10 AM, 3 PM, and 8 PM, each day to match the times of phase activation and suspension. Even though we make one-hour-ahead predictions, our predictions depend on model parameters that are trained using all the data; thus, our phase analysis is, in a sense, retrospective even though predictions are prospective. This model allows us to make probabilistic inference about reaching the conditions for environmental phase alerts in Mexico City and about Mexico City's compliance with MAAQS.

In the missing data context, we suppose that arbitrary $Y_{i t}^{O}$ or $Y_{i t}^{P M}$ is unobserved. This could be due to limited sampling or for model validation on a holdout dataset, but we only take this predictive approach when comparing models in Section 3.3. Each held-out observation is updated or imputed as a part of model fitting using a Gibbs sampler (See Appendix C for details).

To predict future pollution measurements using our model, Equation 1, we use the following formula:

$$
\begin{aligned}
& Y_{i(t+1)}^{O}=\mathbf{x}_{i t}^{T} \boldsymbol{\beta}_{1 i}+\mathbf{L}_{i(t+1)}^{O}{ }^{T} \gamma_{1 i}+\psi_{1 i}+\epsilon_{1 i(t+1)} \\
& Y_{i(t+1)}^{P M}=\mathbf{x}_{i t}^{T} \boldsymbol{\beta}_{2 i}+\mathbf{L}_{i(t+1)}^{P M}{ }^{T} \gamma_{2 i}+\psi_{2 i}+\epsilon_{2 i(t+1)} .
\end{aligned}
$$

Note that one-step-ahead predictions do not rely on future covariates. We use this type of prediction for both inferential tasks (See Sections 4.1 and 4.2).

In our setting, predicted phase alerts come from the one-hour-ahead ozone predictions $\left(\hat{Y}_{i t}^{O}\right)$ and the predicted 24hour average $\mathrm{PM}_{10}$ concentration $\left(\widehat{\bar{Y}_{i t}^{P M}}\right)$, where $\widehat{\bar{Y}_{i t}} \widehat{\text { is }}$ is the average of the 23 most recent observed $\mathrm{PM}_{10}$ concentrations $\left(Y_{i(t-1)}^{P M}, \ldots, Y_{i(t-23)}^{P M}\right)$ and the forecasted $\mathrm{PM}_{10}$ level $\left(\hat{Y}_{i t}^{P M}\right)$. Nationally legislated thresholds depend on 24-hour average $\mathrm{PM}_{10}$ and on 8-hour average $O_{3}$. Similar to $\widehat{\bar{Y}_{i t}} \widehat{ }$, predicted 8-hour average ozone concentration $\left(\widehat{\bar{Y}_{i t}^{O}}\right)$ is an average of a one-hour-ahead prediction and the previous seven ozone measurements $\left(Y_{i(t-1)}^{O}, \ldots, Y_{i(t-7)}^{O}\right)$. For predictions of both $\widehat{\bar{Y}}_{i t}^{P M}$ and $\widehat{\bar{Y}}_{i t}^{O}$ on January 1, 2017, we rely on hourly observations from December 31, 2016.

\section{2 | Posterior Inference}

The primary inferential goal for this dataset is to assess how often the Mexico City metropolitan area (1) is at risk for declaring phase I or II emergencies and (2) exceeds MAAQS. For each task, we take different modeling approaches, as discussed in Section 3.1. To analyze the risk of phase I and II emergencies, we fit the model to all the data. In contrast, when examining pollution level exceedances, we fit the model sequentially. For both tasks, we use one-step-ahead predictions for ozone and $\mathrm{PM}_{10}$ concentrations. These posterior predictions allow us to carry out probabilistic inference on emergency phases and MAAQS exceedances to assess how often the Mexico City metropolitan area was at risk of a pollution emergency and how often pollution levels were unsafe according to Mexican federal guidelines (Diario Oficial de la Federación, 2014a,b).

To define useful quantities, let $j$ index region and $d$ index day, such that each station $i \in j$ and each hour $t \in d$. Additionally, we define ${\overline{Y_{i t}}}^{P M}$ to be the 24-hour running average of $\mathrm{PM}_{10}$ at time $t$ and station $i$. We define the following maxima:

$$
\begin{aligned}
Z_{j t}^{O} & =\max _{i \in j} Y_{i t}^{O} & W_{j d}^{O} & =\max _{t \in d} \max _{i \in j} Y_{i t}^{O} \\
Z_{j t}^{P M} & =\max _{i \in j} \bar{Y}_{i t}^{P M} & W_{j d}^{P M} & =\max _{t \in d} \max _{i \in j} \bar{Y}_{i t}^{P M},
\end{aligned}
$$


where $Z$ 's are regional maxima for any hour $t$ and $W$ 's are daily regional maxima. It is important to clarify that although these maxima often rely on data observed prior to time $t$ or day $d$, these quantities are used to define exceedances at time $t$ or day $d$. There is limited literature about the distributions and properties of maxima for correlated random variables (see Gupta et al., 1985; Ho and Hsing, 1996). However, these examples are too constrained for our application. In the spatial literature, modeling extreme values, and sometimes maxima, is well-studied (see, e.g., Sang and Gelfand, 2009, 2010; Davison et al., 2012); however, these approaches generally invoke generalized extreme value (GEV) distribution models. As noted in Section 2, our inference depends on relatively few maxima over few sites or hours, so GEV theory is not applicable. In fact, using the definitions in (5), we do not model the maxima directly. Instead, we obtain the derived posterior predictive distribution for $Z_{j t}^{O}, Z_{j t}^{P M}, W_{j d}^{O}$, and $W_{j d}^{P M}$ from posterior predictive samples of $Y_{i t}^{O}$ and $Y_{i t}^{P M}$.

The states of Mexico City's phase alert system $S_{j t} \in\{0,1,2\}$ are completely determined by $Z_{j t}^{O}$ and $Z_{j t}^{P M}$ (see Section 1 and Table 1). To obtain the maximum phase alert for a day $d$ in some region $j\left(\max _{t \in d} S_{j t}\right)$, we use $W_{j d}^{O}$ and $W_{j d}^{P M}$. One may also wish to infer the distribution of the highest phase alert in any region on day $d\left(\max _{j} \max _{t \in d} S_{j t}\right)$. All these derived posterior quantities can be obtained after model fitting. Using derived posterior predictive distributions for various maxima, as well as associated phase states and threshold exceedances, we can compute hourly and daily probabilities of (possible) phase alerts and pollution exceedances regionally and city-wide. The utility of these probabilities is the insight they can provide regarding how often the Mexico City metropolitan area is at risk of a pollution emergency, even if phase alerts were not enacted due to meteorological forecasts.

Inference for the first task, analysis of the phase emergencies, requires analysis of regional pollution levels at 10 AM, 3 PM, and 8 PM. Specifically, phase states depend on maxima of stations over regions. If we carry out inference on a daily scale, double maxima are needed, maxima over hours and stations within regions. Because phase alerts are based upon one-hour ozone measurements and 24-hour average PM 10 (Administración Pública de la Ciudad de México, 2016), we predict these averages as described in Section 3.1. These predictions allow us to compute predictions for derived quantities $\widehat{Z_{j t}^{O}}, \widehat{Z_{j t}^{P M}}, \widehat{W_{j d}^{O}}$, and $\widehat{W_{j d}^{P M}}$, which in turn define phase state predictions $\widehat{S_{j t}}$. Again, inference on phase predictions is our primary goal.

We also carry out similar inference on MAAQS exceedance for ozone and $\mathrm{PM}_{10}$. Again, we are interested in regional (i.e. stations within a specified region) and city-level (i.e. at any station in the city) exceedance, hourly and daily. Similar, but not identical to phase alerts, inference for pollution exceedances relies upon maxima of one-hour ozone, eight-hour average ozone ${\overline{Y_{i t}}}^{O}$, and 24-hour average $\mathrm{PM}_{10}$. For eight-hour average ozone, we define $Z_{j t}^{\bar{O}}$ to be the regional maxima at time $t$ and $W_{j d}^{\bar{O}}$ to be the daily maxima for region $j$. In this case, thresholds are much lower than the thresholds Atmospheric Environmental Contingency Program in Mexico City (Diario Oficial de la Federación, 2014a,b; Administración Pública de la Ciudad de México, 2016). Because nationally legislated ozone and PM 10 thresholds were specified to avoid reaching unsafe pollution levels, comparisons to MAAQS indicate how often Mexico City reaches unsafe pollution levels without triggering any city protocols. Such comparisons highlight important differences in how pollution emergencies are defined in Mexico City relative to nationally legislated levels.

\section{3 | Model Selection}

In this subsection, we describe the model selection process leading to the model under which we carry out all the inference described in the previous subsections. Our model selection decision centers around answering how many and which lagged terms should be used in our spatiotemporal model. In our exploratory analyses, we argued that the variance of ozone and $\mathrm{PM}_{10}$ vary with the time of day and time of year. We indicated that this could be remedied in one of two ways: (1) using variance-stabilizing transformations to address correlation between mean and variance in the data or (2) modeling the heteroscedasticity directly. For transformation approaches, we consider modeling the data on 
different scales (truncated, log, and square root) to stabilize the mean-variance correlation. To answer these modeling questions, we hold out $10 \%$ of both pollutants and treat these as missing data. Specifically, the locations and times of the hold-out data are selected at random, and both ozone and $\mathrm{PM}_{10}$ are held-out at these location-time pairs so that model comparison can be made using joint predictions. We make predictions at these held-out observations and compare competing models based on several criteria: predictive mean squared error $\left(E\left(Y_{i} \mid \mathbf{Y}_{o b s}\right)-y_{i}\right)^{2}$ (PMSE) or mean absolute error $\left|E\left(Y_{i} \mid \mathrm{Y}_{\text {obs }}\right)-y_{i}\right|(\mathrm{PMAE}), 100 \times \alpha \%$ prediction interval coverage, and continuous rank probability scores (CRPS) (Gneiting and Raftery, 2007), where

$$
\operatorname{CRPS}\left(F_{i}, y_{i}\right)=\int_{-\infty}^{\infty}\left(F_{i}(x)-\mathbf{1}\left(x \geq y_{i}\right)\right)^{2} d x=\mathbf{E}\left|Y_{i}-y_{i}\right|-\frac{1}{2} \mathbf{E}\left|Y_{i}-Y_{i^{\prime}}\right| .
$$

Because we are utilizing MCMC to fit our model, we use posterior predictive samples for a Monte Carlo approximation of CRPS using an empirical CDF approximation (see, e.g., Krüger et al., 2016),

$$
\operatorname{CRPS}\left(\hat{F}_{i}^{\mathrm{ECDF}}, y_{i}\right)=\frac{1}{M} \sum_{j=1}^{M}\left|Y_{j}-y_{i}\right|-\frac{1}{2 M^{2}} \sum_{j=1}^{M} \sum_{k=1}^{M}\left|Y_{j}-Y_{k}\right|,
$$

where $M$ is the number of MCMC samples used, $Y_{i}$ are predictions, and $y_{i}$ are observed values. We then average $\operatorname{CRPS}\left(\hat{F}_{i}^{\mathrm{ECDF}}, y_{i}\right)$ over all held-out data. In addition to being a proper scoring rule (Gneiting and Raftery, 2007), because CRPS considers how well the entire predictive distribution matches the observed data rather than only the predictive mean (MAE and MSE) or quantiles (prediction interval coverage), we prefer it as selection criterion. For multivariate predictions, as we have in this analysis, we consider the energy score (ES), which is a multivariate generalization of CRPS. For a set of multivariate predictions $\mathrm{Y}$, ES is defined as

$$
\mathrm{ES}(P, \mathbf{y})=\frac{1}{2} E_{P}\left\|\mathbf{Y}-\mathbf{Y}^{\prime}\right\|^{\beta}-E_{P}\|\mathbf{Y}-\mathbf{y}\|^{\beta}
$$

where $\mathrm{y}$ is an observation, $\beta \in(0,2)$, and $P$ is a probability measure (Gneiting and Raftery, 2007). It is common to fix $\beta=1$ (see, e.g., Gneiting et al., 2008; Jordan et al., 2017). For a set of $M M C M C$ predictions $Y=Y_{1}, \ldots, Y_{M}$ for a held-out observation y, the empirical ES reduces to

$$
\mathrm{ES}(\mathbf{Y}, \mathbf{y})=\frac{1}{M} \sum_{j=1}^{M}\left\|\mathbf{Y}_{j}-\mathbf{y}\right\|-\frac{1}{2 M^{2}} \sum_{i=1}^{M} \sum_{j=1}^{M}\left\|\mathbf{Y}_{i}-\mathbf{Y}_{j}\right\|
$$

as was discussed in Gneiting et al. (2008). Energy scores are scale-sensitive, meaning that if one of the variables has a much larger scale than other of the variables, it dominates the norms in Equation 9. In our data, $\mathrm{PM}_{10}$ concentration in $\mu \mathrm{g} / \mathrm{m}^{3}$ takes values larger than ozone in $\mathrm{ppb}$. To assure that predictions for each pollutant are similarly weighted, we standardize the predictions and hold-out values for each pollutant (i.e. subtract the sample mean and divide by the sample standard deviation). Like CRPS, we average ES over all held-out data.

Interestingly, the heteroscedastic models with variance that varies over hour of the day and month of the year performed uniformly worse than homoscedastic counterparts that used VST's to stabilize the mean-variance correlation. Testing various combinations of square-root transformations, log transformations, and truncated distributions, we found that models using the square-root transformation for ozone and the log transformation for $\mathrm{PM}_{10}$ gave the best predictive performance. So, for model selection, we only give the results for six models which use the square-root transformation for ozone and the log-transformation for $\mathrm{PM}_{10}$ but differ in terms of which lags are included in the 
model. The results of this comparison are given in Table 3.

\begin{tabular}{lllrrrrrrrrr}
\hline & & & $\mathrm{O}_{3}$ & $\mathrm{O}_{3}$ & $\mathrm{O}_{3}$ & $\mathrm{O}_{3}$ & $\mathrm{PM}_{10}$ & $\mathrm{PM}_{10}$ & $\mathrm{PM}_{10}$ & $\mathrm{PM}_{10}$ \\
\hline & Lags & ES & $\mathrm{CRPS}$ & $\mathrm{RMSE}$ & $\mathrm{MAE}$ & $\mathrm{Cov}$ & $\mathrm{CRPS}$ & $\mathrm{RMSE}$ & $\mathrm{MAE}$ & $\mathrm{Cov}$ \\
\hline 1 & $(1,2)$ & 0.2552 & 2.5392 & 5.0448 & 3.3409 & 0.8867 & 6.7263 & 14.5427 & 8.7725 & 0.9228 \\
\hline 2 & $(1,2,24)$ & 0.2513 & 2.5158 & 4.9709 & 3.3035 & 0.8925 & 6.6176 & 14.1469 & 8.6189 & 0.9217 \\
\hline 3 & $(1,2,24,168)$ & 0.2505 & 2.5140 & 4.9614 & 3.2982 & 0.8941 & 6.5947 & 14.0922 & 8.6285 & 0.9229 \\
\hline 4 & $(1,2,12)$ & 0.2540 & 2.5298 & 5.0274 & 3.3244 & 0.8887 & 6.6959 & 14.4314 & 8.7864 & 0.9230 \\
\hline 5 & $(1,2,12,24)$ & 0.2509 & 2.5168 & 4.9726 & 3.3115 & 0.8917 & 6.6035 & 14.0981 & 8.6296 & 0.9220 \\
\hline 6 & $(1,2,12,24,168)$ & 0.2507 & 2.5154 & 4.9651 & 3.2993 & 0.8941 & 6.5976 & 14.0972 & 8.6378 & 0.9225 \\
\hline
\end{tabular}

TAB LE 3 Predictive model comparison. The "Lags" label indicates which lags are used for both outcomes. "ES," "CRPS," "MSE," "MAE," and "Cov" head columns giving ES, CRPS, MSE, MAE, and 90\% prediction interval coverage. Best performances are indicated with bold text.

We further note that in preliminary modeling, we found that models which included a lag-3 and other higher order lags or that excluded lag-2 saw no improvement in terms of prediction; thus, we arrived at the models included in Table 3. Given these results, we argue that the best model for ozone and $\mathrm{PM}_{10}$ uses lags 1, 2, 24, and 168. So, the ensuing results are presented for this model.

\section{4 | RESULTS AND DISCUSSION}

We present our inference based on a joint model for ozone and $\mathrm{PM}_{10}$ with four lags (1, 2, 24, and 168). We use a Gibbs sampler to obtain 100,000 posterior samples after a burn-in of 10,000 iterations. Posterior parameter inference is discussed in the online supplement, and these results validate many of the modeling decisions suggested by our exploratory analysis in Section 2 and discussed in Section 3. Because we have $N=210240$ observations, the predictive space is large $\left(2 \times N \approx 4 \times 10^{5}\right)$. Thus, we thin the posterior predictive samples using every $10^{\text {th }}$ sample. By thinning, we make 10,000 roughly independent predictions. These predictions are used to carry out analyses in Sections 4.1 and 4.2.

\subsection{Analysis of the Phase Alert System}

In this section, we analyze Mexico City's phase alert system to identify when the Mexico City metropolitan area was predicted to be at risk for pollution emergencies. For this, we use one-hour-ahead predictions for pollution levels each day at the three decision times reference in Section 1: 10 AM, 3 PM, and 8 PM. Thus, our analysis predicts at three hours per day, altogether 1095 hours in 2017. This allows us to assess probabilities of the risk of phase alerts given the most recent weather conditions and pollution levels. Again, we note that the risk of a phase alert is not the same as a phase alert. As discussed above, we use parameter values trained on the entire dataset which enables effective prediction in early months. Because the pollutant thresholds for triggering phase alerts are very high, most of the year has very low probabilities for phase activation. In May of 2017, however, Mexico City was featured prominently in the news for having dangerously high ozone levels which led to an activation of a phase I pollution emergency. Phase probabilities aggregated over regions $\left(P\left(\max _{j} S_{j d}=k\right)\right.$ for state $k$ ) are displayed in Figure 3. Regional phase I probabilities for each day $\left(P\left(S_{j d}=k\right)\right.$ for phase $\left.k\right)$ are given in Figure 4. In Figure 4, we do not show phase II probabilities because they are so 
low. Additionally, we only display region NE compared to other regions because all other regions overlap (See Figure 4). Both plots (Figures 3 and 4) show high probabilities (> 1/2) of phase I activation from May 16th to May 25, coinciding with the time of the actually declared phase I emergency. Because this phase I alert was triggered by ozone levels, the emergency was declared city-wide, as indicated by the agreement of regional curves in Figure 4.

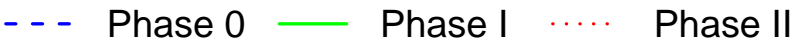

Daily 2017 Phase Probabilities

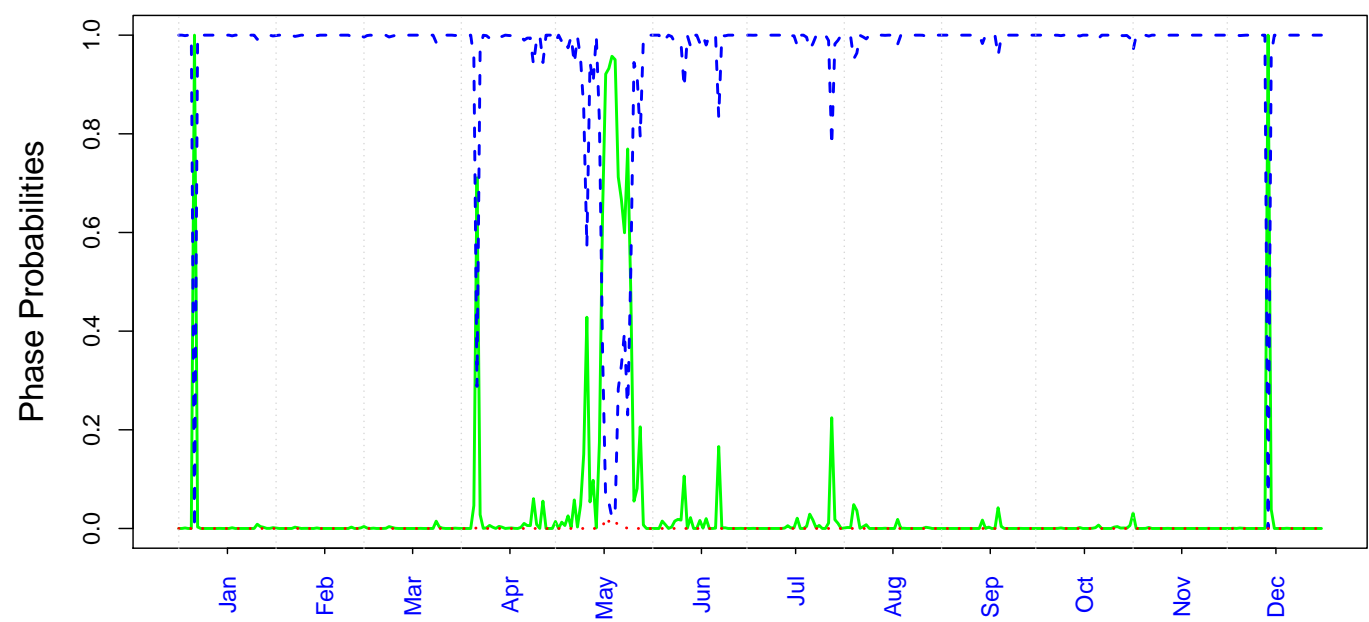

FIGURE 3 Phase probabilities in Mexico City, aggregated over all regions.

Daily Regional 2017 Phase I Probabilities

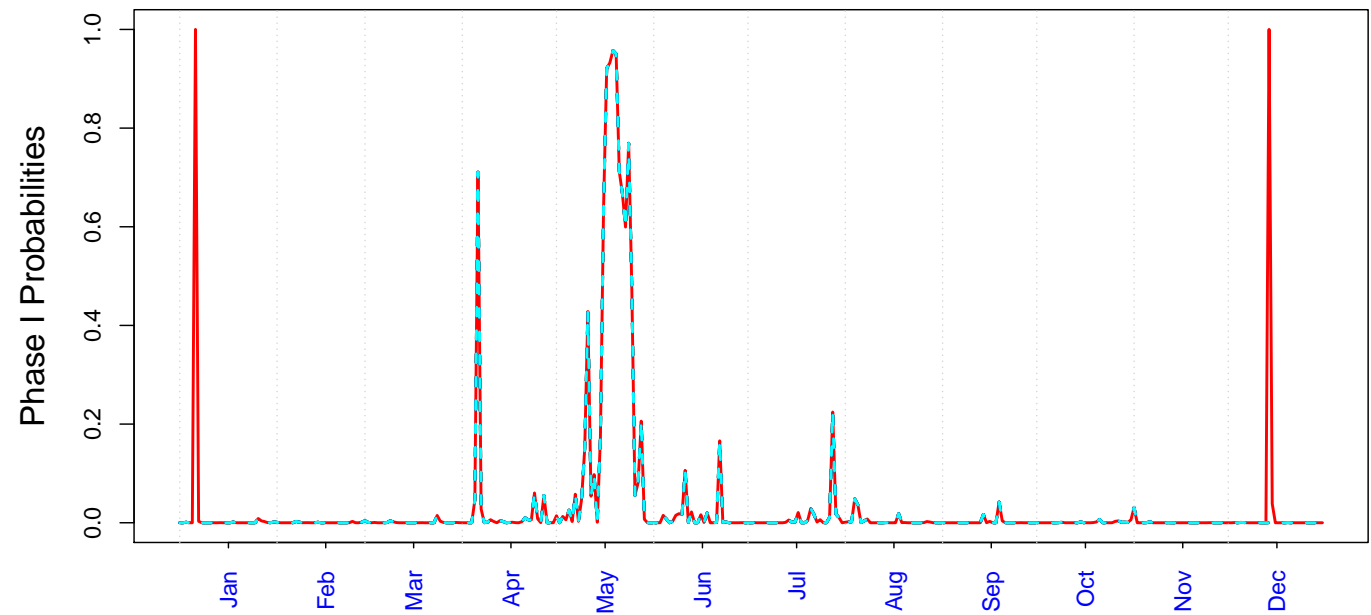

FIGURE 4 Daily phase I probabilities for Mexico City over the year by region. Phase II probabilities are not included because they are uniformly low.

On April 6th, predicted ozone levels were sufficient to trigger a phase I emergency city-wide, although a phase alert was not declared. On only two occasions, one in January and one in December, was any region at risk of activating the emergency contingency plan due to $\mathrm{PM}_{10}$ levels. These high phase I probabilities were limited to the northeast region 
(See the red peaks in Figure 4). Again, it is worth noting that a phase I alert triggered by $\mathrm{PM}_{10}$ corresponds to $\mathrm{PM}_{10}$ levels that are nearly three times the levels specified as safe by Mexican legislation (Diario Oficial de la Federación, 2014b).

In Table 4, we provide posterior means and 95\% credible intervals for the number of hours and days for which the Mexico City metropolitan area is at risk for pollution emergencies $\left(\sum_{d} \mathbf{1}\left(S_{j d}=k\right)\right.$ for phase $\left.k\right)$. The first thing to notice is that there are very few hours and days when the metropolitan area or its sub-regions are at risk of pollution emergencies. Note that the posterior predictive mean for risk of a pollution emergency is 11 days and 11 hours for the central, northwest, southeast, and southwest regions. These counts are not necessarily reflective of conditions in central and northwest regions. Instead, these counts are indicative of predicted city-wide phase I alerts due to predicted ozone exceedances in the southeast and southwest regions, one in April and 10 in May (see Figures 3 and 4). The northeast region is the only region that had more average predicted hours and days of pollution emergency than other regions. We predict six hours of risk for phase I emergencies in the northeast region due to $\mathrm{PM}_{10}$ levels over two non-consecutive days, one day in January and one in December. Because the northeast region was the only region where a predicted phase alert was triggered by $\mathrm{PM}_{10}$, the predicted risk of a phase alert was limited to the northeast region. No phase alert was declared even though predicted phase probabilities were equal to one. Thus, the reason for not declaring an emergency must be attributed to meteorological conditions. While we do know the exact rationale for not declaring a phase emergency, we speculate that the emergency was not declared because these predicted phase risks were transient, lasting only one day each.

\begin{tabular}{|l|rrrrrr|}
\hline & \multicolumn{7}{|c|}{ Hours (total of 1095 || 3 hours / day) } \\
& CE & NE & NW & SE & SW & Any \\
\hline No Phase & $1084 \pm 4$ & $1078 \pm 4$ & $1084 \pm 4$ & $1084 \pm 4$ & $1084 \pm 4$ & $1078 \pm 4$ \\
\hline Phase I & $11 \pm 4$ & $17 \pm 4$ & $11 \pm 4$ & $11 \pm 4$ & $11 \pm 4$ & $17 \pm 4$ \\
\hline Phase II & $0.09(0,1)$ & $0.09(0,1)$ & $0.09(0,1)$ & $0.09(0,1)$ & $0.09(0,1)$ & $0.09(0,1)$ \\
\hline & & & Days (total of 365) & & \\
\hline No Phase & $354 \pm 4$ & $352 \pm 4$ & $354 \pm 4$ & $354 \pm 4$ & $354 \pm 4$ & $352 \pm 4$ \\
\hline Phase I & $11 \pm 4$ & $13 \pm 4$ & $11 \pm 4$ & $11 \pm 4$ & $11 \pm 4$ & $13 \pm 4$ \\
\hline Phase II & $0.09(0,1)$ & $0.09(0,1)$ & $0.09(0,1)$ & $0.09(0,1)$ & $0.09(0,1)$ & $0.09(0,1)$ \\
\hline
\end{tabular}

TA B LE 4 One-hour-ahead posterior predictive estimates for the (Top) Number of hours in each phase state for each region (Bottom) Number of days for which that phase state was attained (the maxima attained each day). Posterior means and $95 \%$ credible intervals are given for each region, using \pm or parentheses. The "Any" label indicates that this is the maximum across regions.

\subsection{Comparison of Mexico City to Mexican Legislated Thresholds}

In this section, we examine the probability that maxima within regions exceed MAAQS on a given day $\left(W_{j d}^{O}\right.$ and $W_{j d}^{P M}$ from Section 3.2). In contrast to phase alert probabilities, which are generally very low, exceedance probabilities are often high through much of the year. Because MAAQS are more reflective of healthy levels of ozone and PM 10 , comparison between the exceedance probabilities and emergency phase probabilities highlights how often Mexico City has harmful pollution levels without triggering phase alerts. Additionally, this analysis gives insight into the probability of triggering phase alerts in Mexico City if MAAQS were adopted for Mexico City's Atmospheric Environmental 
Contingency Program. For our purposes, we group either type of ozone exceedance, one or eight-hour, together. In the online supplement, we focus on three months, April, August, and December, to illustrate how exceedance probabilities change over the course of the year.

We continue the prospective analysis for all months except January, fitting the model up until the last hour of the previous month to predict pollution exceedance for the month of interest. Because we fit the model sequentially, prospective predictions for January are poor because the model has not been trained on data for these times. Using these predictions, we give posterior means and $95 \%$ credible intervals for the one-hour-ahead predicted proportion of hours and days of exceedance for each region (i.e. $P\left(Z_{j t}^{O}>95 \mathrm{ppb} \cup Z_{j t}^{\bar{O}}>70 \mathrm{ppb}\right), P\left(Z_{j t}^{P M}>75 \mu g / \mathrm{m}^{3}\right), P\left(W_{j d}^{O}>\right.$ $\left.95 \mathrm{ppb} \cup W_{j d}^{\bar{O}}>70 \mathrm{ppb}\right)$, and $P\left(W_{j d}^{P M}>75 \mu \mathrm{g} / \mathrm{m}^{3}\right)$, as defined in Section 3.2). The results for ozone are given in Table 5, and the estimates for $\mathrm{PM}_{10}$ are presented in Table 6. For ozone, the proportion of exceedances in both hours and days decreases as latitude increases, with northern regions showing nearly half as many exceedances as the southern regions, on average. The trend for $\mathrm{PM}_{10}$ is less clear, although there is significant variability across regions. The northeast region has many more predicted $\mathrm{PM}_{10}$ exceedances than any other region. This is due to the large industrial economy located within this region. By contrast, the southwest region has, comparatively, very few $\mathrm{PM}_{10}$ exceedances.

\begin{tabular}{|c|c|c|c|c|c|c|c|c|c|c|c|c|}
\hline \multirow[t]{2}{*}{ Ozone } & \multicolumn{6}{|c|}{ Hours (total of 8016 ) } & \multicolumn{6}{|c|}{ Days (total of 334) } \\
\hline & CE & NE & NW & SE & SW & Any & CE & NE & NW & SE & SW & Any \\
\hline Mean & 0.147 & 0.066 & 0.093 & 0.194 & 0.186 & 0.252 & 0.651 & 0.367 & 0.499 & 0.671 & 0.696 & 0.794 \\
\hline $2.5 \%$ & 0.143 & 0.064 & 0.090 & 0.190 & 0.183 & 0.249 & 0.626 & 0.338 & 0.467 & 0.647 & 0.674 & 0.773 \\
\hline $97.5 \%$ & 0.150 & 0.069 & 0.096 & 0.197 & 0.189 & 0.256 & 0.677 & 0.395 & 0.530 & 0.698 & 0.719 & 0.814 \\
\hline
\end{tabular}

TAB LE 5 One-hour-ahead posterior predictive estimates for the (Left) Proportion of hours where either of the Mexican legislated ozone limits (one-hour or eight-hour) were exceeded (Right) Proportion of days where either of the Mexican legislated ozone limits were exceeded. Posterior means and $95 \%$ credible intervals are given for each region. The "Any" label indicates that at least one region has an exceedance for one or more location for the time level (hour or day).

\begin{tabular}{|c|c|c|c|c|c|c|c|c|c|c|c|c|}
\hline \multirow[t]{2}{*}{$\mathrm{PM}_{10}$} & \multicolumn{6}{|c|}{ Hours (total of 8016) } & \multicolumn{6}{|c|}{ Days (total of 334) } \\
\hline & CE & NE & NW & SE & SW & Any & CE & $\mathrm{NE}$ & NW & SE & sW & Any \\
\hline Mean & 0.123 & 0.408 & 0.221 & 0.247 & 0.0112 & 0.429 & 0.218 & 0.5223 & 0.333 & 0.370 & 0.026 & 0.535 \\
\hline $2.5 \%$ & 0.122 & 0.406 & 0.219 & 0.245 & 0.0106 & 0.427 & 0.210 & 0.512 & 0.323 & 0.362 & 0.021 & 0.524 \\
\hline $97.5 \%$ & 0.125 & 0.410 & 0.223 & 0.249 & 0.0119 & 0.430 & 0.228 & 0.533 & 0.341 & 0.377 & 0.030 & 0.545 \\
\hline
\end{tabular}

TA B LE 6 One-hour-ahead posterior predictive estimates for the (Left) Proportion of hours where the Mexican legislated 24-hour PM 10 limits were exceeded (Right) Proportion of days where either of the Mexican legislated PM 10 limits were exceeded. The "Any" label indicates that at least one region has an exceedance for one or more location for the time level (hour or day).

Lastly, we discuss the proportion of predicted hourly exceedances as a function of the month of the year and of the hour of the day. The summaries for ozone by month and hour-of-day are plotted in Figure 5, while we display PM 10 exceedances only by month (Figure $5 b$ ). We do not plot $\mathrm{PM}_{10}$ exceedances as function of the hour of the day because $\mathrm{PM}_{10}$ exceedances depend on 24-hour averages; thus, trends over time-of-day are not meaningful. As a function of month, the patterns of ozone and $\mathrm{PM}_{10}$ exceedances are clear. For ozone, the proportion of exceedances reaches a peak in May and is high in March, April, and June. We attribute these high ozone levels to warm times of the year that are dry compared to the rainy season (June-August). $\mathrm{PM}_{10}$ exceedance appears to co-vary strongly with the rainy season as well, which is captured by relative humidity in our model. In particular, June, July, August, and September have almost 
no exceedances for $\mathrm{PM}_{10}$. The coldest months (December and February) have higher probabilities of $\mathrm{PM}_{10}$ exceedance than warmer months that are similarly dry. Mexico City's pollution output is higher during winter festivities like Our Lady of Guadalupe, Christmas, and New Year due to fireworks and increased motor traffic. In conjunction with increased pollution output, pollution exceedances in cold months are also due to thermal inversion that traps pollution in the Valley of Mexico where Mexico City lies. Ozone exceedances also tend to peak in the afternoon to evening. Because ozone levels can exceed thresholds for either one-hour or eight-hour average ozone, we expect two peaks in ozone exceedance as a function of hour. The one-hour peak occurs around $4 \mathrm{PM}(16: 00)$ when the temperature is highest. The peak of eight-hour average ozone peaks around 7 or 8 PM (19:00 or 20:00), after eight hours of relatively high ozone levels. These peaks can be seen in Figure $5 c$.

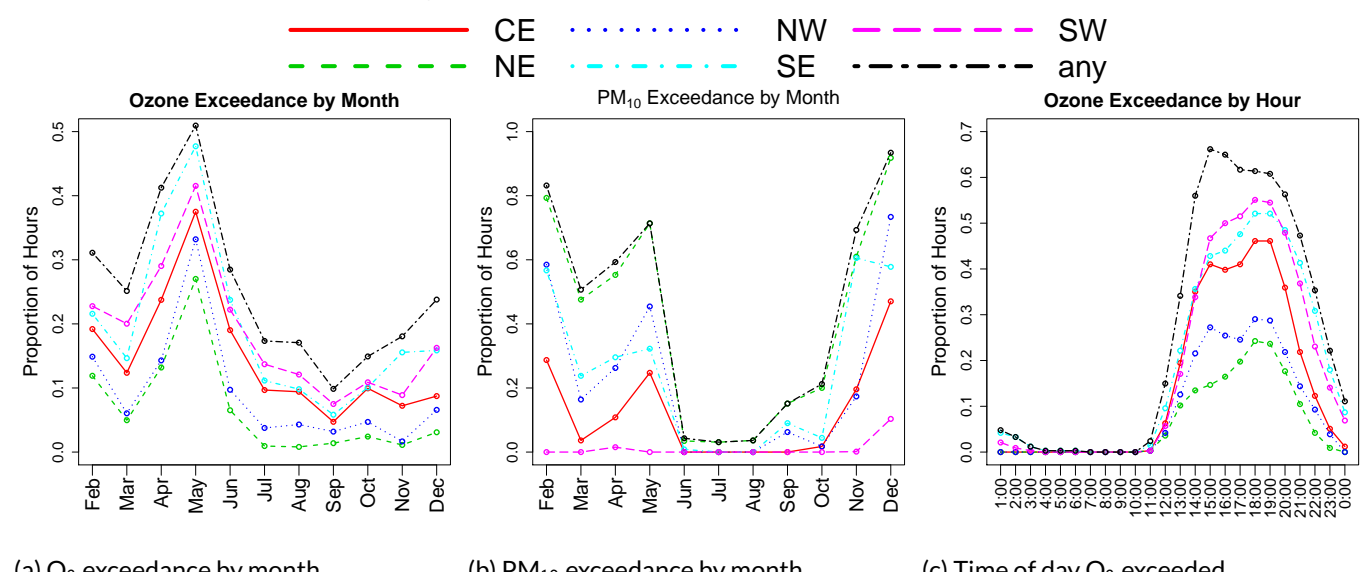

(a) $\mathrm{O}_{3}$ exceedance by month

(b) $\mathrm{PM}_{10}$ exceedance by month

(c) Time of day $\mathrm{O}_{3}$ exceeded

FIGURE 5 Posterior predictive means for the proportion of hours of exceedance as function of month and hour of the day.

\section{5 | CONCLUSIONS AND FUTURE WORK}

We have discussed the monitoring network for ozone and $\mathrm{PM}_{10}$ within Mexico City and proposed a joint spatiotemporal model for ozone and $\mathrm{PM}_{10}$ concentrations. This model was used to predict future pollutant concentrations. Our predictions were then used to obtain derived distributions for regional maxima of ozone and $\mathrm{PM}_{10}$ which are needed to determine Mexico City's pollution emergency phases. Additionally, our predictions are used to assess compliance with MAAQS. We find that predicted risk of pollution emergency is rare and are predicted for only a few periods of 2017. By contrast, we demonstrate that predicted exceedance of Mexico's ambient air quality standards is common.

In future work, we will attempt to operationalize our model so that it can be used in practice. This would require real time (hourly) fitting of the model as new measurements are available. Our modeling is amenable to sequential updating as well as possible parallelization though considerable optimization remains before this could be implemented in practice. Once implemented, our model could warn of potential pollution emergencies or compliance issues, allowing regional and city-wide adjustments, warnings, responses, and decision-making to be made earlier. Our model could incorporate weather forecasts to perhaps more accurately forecast pollutant levels farther ahead than our one-hourahead predictions. 


\section{REFERENCES}

101st United States Congress (1990) Clean Air Act Amendments of 1990. Public Law 101-549. 104 Stat. 2399.

Administración Pública de la Ciudad de México (2016) Órgano de difusión del gobierno de la ciudad de méxico.

Angle, R. and Sandhu, H. (1989) Urban and rural ozone concentrations in alberta, canada. Atmospheric Environment (1967), 23, 215-221.

Banerjee, S., Carlin, B. P. and Gelfand, A. E. (2014) Hierarchical modeling and analysis for spatial data. CRC Press.

Barraza-Villarreal, A., Sunyer, J., Hernandez-Cadena, L., Escamilla-Nuñez, M. C., Sienra-Monge, J. J., Ramírez-Aguilar, M., Cortez-Lugo, M., Holguin, F., Diaz-Sánchez, D. and Olin, A. C. (2008) Air pollution, airway inflammation, and lung function in a cohort study of mexico city schoolchildren. Environmental health perspectives, 116, 832.

Bell, M. L., Peng, R. D. and Dominici, F. (2006) The exposure-response curve for ozone and risk of mortality and the adequacy of current ozone regulations. Environmental health perspectives, 114, 532.

Berrocal, V. J., Gelfand, A. E. and Holland, D. M. (2010) A spatio-temporal downscaler for output from numerical models. Journal of agricultural, biological, and environmental statistics, 15, 176-197.

Bravo-Alvarez, H. and Torres-Jardón, R. (2002) Air pollution levels and trends in the mexico city metropolitan area. In Urban Air Pollution and Forests, 121-159. Springer.

Brunekreef, B. and Holgate, S. T. (2002) Air pollution and health. The lancet, 360, 1233-1242.

Clements, N., Piedrahita, R., Ortega, J., Peel, J. L., Hannigan, M., Miller, S. L. and Milford, J. B. (2012) Characterization and nonparametric regression of rural and urban coarse particulate matter mass concentrations in northeastern colorado. Aerosol Science and Technology, 46, 108-123.

Cocchi, D., Greco, F. and Trivisano, C. (2007) Hierarchical space-time modelling of pm10 pollution. Atmospheric environment, 41, 532-542.

Davis, L. W. (2017) Saturday driving restrictions fail to improve air quality in mexico city. Scientific Reports, 7, 41652.

Davison, A. C., Padoan, S. A. and Ribatet, M. (2012) Statistical modeling of spatial extremes. Statistical science, 27, $161-186$.

Departamento del Distrito Federal, Gobierno del Estado de México, Secretaría de Recursos Naturales y Pesca and Secretaría de Salud (1996) Programa para mejorar la calidad del aire en el valle de mexico 1995-2000.

Diario Oficial de la Federación (2014a) Norma Oficial Mexicana NOM-020-SSA1-2014.

- (2014b) Norma Oficial Mexicana NOM-025-SSA1-2014.

Dueñas, C., Fernández, M., Canete, S., Carretero, J. and Liger, E. (2004) Analyses of ozone in urban and rural sites in málaga (spain). Chemosphere, 56, 631-639.

European Environment Agency (2016) Exceedance of air quality limit values in urban areas.

Gelfand, A. E., Kim, H.-J., Sirmans, C. and Banerjee, S. (2003) Spatial modeling with spatially varying coefficient processes. Journal of the American Statistical Association, 98, 387-396.

Gelman, A., Carlin, J. B., Stern, H. S., Dunson, D. B., Vehtari, A. and Rubin, D. B. (2014) Bayesian data analysis, vol. 2. CRC press Boca Raton, FL.

Gneiting, T. and Raftery, A. E. (2007) Strictly proper scoring rules, prediction, and estimation. Journal of the American Statistical Association, 102, 359-378. 
Gneiting, T., Stanberry, L. I., Grimit, E. P., Held, L. and Johnson, N. A. (2008) Assessing probabilistic forecasts of multivariate quantities, with an application to ensemble predictions of surface winds. Test, 17, 211.

Gouveia, N., Junger, W. L., Romieu, I., Cifuentes, L. A., de Leon, A. P., Vera, J., Strappa, V., Hurtado-Díaz, M., Miranda-Soberanis, V. and Rojas-Bracho, L. (2018) Effects of air pollution on infant and children respiratory mortality in four large latin-american cities. Environmental Pollution, 232, 385-391.

Grzebyk, M. and Wackernagel, H. (1994) Multivariate analysis and spatial/temporal scales: real and complex models. In Proceedings of the XVIIth International Biometrics Conference, vol. 1, 19-33. Citeseer.

Gupta, S. S., Panchapakesan, S. and Sohn, J. K. (1985) On the distribution of the studentized maximum of equally correlated normal random variables. Communications in Statistics-Simulation and Computation, 14, 103-135.

Heal, M. R. and Hammonds, M. D. (2014) Insights into the composition and sources of rural, urban and roadside carbonaceous pm10. Environmental science \& technology, 48, 8995-9003.

Hernández-Garduño, E., Pérez-Neria, J., Paccagnella, A. M., Piña-García, M. A., Munguía-Castro, M., Catalán-Vázquez, M. and Rojas-Ramos, M. (1997) Air pollution and respiratory health in mexico city. Journal of occupational and environmental medicine, 39, 299-307.

Ho, H.-C. and Hsing, T. (1996) On the asymptotic joint distribution of the sum and maximum of stationary normal random variables. Journal of applied probability, 33, 138-145.

Hoek, G., Krishnan, R. M., Beelen, R., Peters, A., Ostro, B., Brunekreef, B. and Kaufman, J. D. (2013) Long-term air pollution exposure and cardio-respiratory mortality: a review. Environmental Health, 12, 43.

Huang, G., Lee, D. and Scott, E. M. (2018) Multivariate space-time modelling of multiple air pollutants and their health effects accounting for exposure uncertainty. Statistics in medicine, 37, 1134-1148.

Instituto Nacional de Ecología y Cambio Climático (INECC) (2017) Informe Nacional de Calidad del Aire 2016, México. Coordinación General de Contaminación y Salud Ambiental, Dirección de Investigación sobre la Calidad del Aire y los Contaminantes Climáticos. Ciudad de México.

Jordan, A., Krüger, F. and Lerch, S. (2017) Evaluating probabilistic forecasts with the $r$ package scoringrules. arXiv preprint arXiv:1709.04743.

Kahle, D. and Wickham, H. (2013) ggmap: Spatial visualization with ggplot2. The R Journal, 5, 144-161.

Krüger, F., Lerch, S., Thorarinsdottir, T. L. and Gneiting, T. (2016) Probabilistic forecasting and comparative model assessment based on markov chain monte carlo output. arXiv preprint arXiv:1608.06802.

Lippmann, M. (1989) Health effects of ozone a critical review. Japca, 39, 672-695.

Loomis, D., Castillejos, M., Gold, D. R., McDonnell, W. and Borja-Aburto, V. H. (1999) Air pollution and infant mortality in mexico city. Epidemiology, 118-123.

Loomis, D., Grosse, Y., Lauby-Secretan, B., El Ghissassi, F., Bouvard, V., Benbrahim-Tallaa, L., Guha, N., Baan, R., Mattock, H. and Straif, K. (2013) The carcinogenicity of outdoor air pollution. The lancet oncology, 14, 1262-1263.

Mage, D., Ozolins, G., Peterson, P., Webster, A., Orthofer, R., Vandeweerd, V. and Gwynne, M. (1996) Urban air pollution in megacities of the world. Atmospheric Environment, 30, 681-686.

Matheron, G. (1982) Pour une analyse krigeante des données régionalisées. Centre de Géostatistique, Report N-732, Fontainebleau.

Pope III, C. A. and Dockery, D. W. (2006) Health effects of fine particulate air pollution: lines that connect. Journal of the air \& waste management association, 56, 709-742. 
Riojas-Rodríguez, H., Álamo-Hernández, U., Texcalac-Sangrador, J. L. and Romieu, I. (2014) Health impact assessment of decreases in pm10 and ozone concentrations in the mexico city metropolitan area: A basis for a new air quality management program. salud pública de méxico, 56, 579-591.

Rodríguez, S., Huerta, G. and Reyes, H. (2016) A study of trends for mexico city ozone extremes: 2001-2014. Atmósfera, 29, 107-120.

Romieu, I., Meneses, F., Ruiz, S., Sienra, J. J., Huerta, J., White, M. C. and Etzel, R. A. (1996) Effects of air pollution on the respiratory health of asthmatic children living in mexico city. American journal of respiratory and critical care medicine, 154 , 300-307.

Rue, H. and Held, L. (2005) Gaussian Markov random fields: theory and applications. CRC press.

Sahu, S. K., Gelfand, A. E. and Holland, D. M. (2007) High-resolution space-time ozone modeling for assessing trends. Journal of the American Statistical Association, 102, 1221-1234.

Salam, M. T., Millstein, J., Li, Y.-F., Lurmann, F. W., Margolis, H. G. and Gilliland, F. D. (2005) Birth outcomes and prenatal exposure to ozone, carbon monoxide, and particulate matter: results from the children's health study. Environmental health perspectives, 113, 1638 .

Sang, H. and Gelfand, A. E. (2009) Hierarchical modeling for extreme values observed over space and time. Environmental and ecological statistics, 16, 407-426.

- (2010) Continuous spatial process models for spatial extreme values. Journal of agricultural, biological, and environmental statistics, 15, 49-65.

Sillman, S. (1999) The relation between ozone, nox and hydrocarbons in urban and polluted rural environments. Atmospheric Environment, 33, 1821-1845.

Wackernagel, H. (1994) Cokriging versus kriging in regionalized multivariate data analysis. Geoderma, 62, 83-92.

Weschler, C. J. (2006) Ozone's impact on public health: contributions from indoor exposures to ozone and products of ozoneinitiated chemistry. Environmental health perspectives, 114, 1489.

Zavala, á., Herndon, S., Wood, E., Onasch, T., Knighton, W., Marr, L. C., Kolb, C. and Molina, L. (2009) Evaluation of mobile emissions contributions to mexico city's emissions inventory using on-road and cross-road emission measurements and ambient data. Atmospheric Chemistry and Physics, 9, 6305-6317.

\section{A | FULL CONDITIONAL DISTRIBUTIONS FOR AR MODEL}

We give the full conditional distributions for the model specified in Section 3. We give some additional details here to clarify model fitting. Because $\mathbf{V}_{1}$ and $\mathbf{V}_{2}$ are independent a priori, the joint prior distribution for $\mathbf{V}_{1}$ and $\mathbf{V}_{2}$ is

$$
\left[\mathbf{V}_{1}, \mathbf{V}_{2}\right] \propto \exp \left(-\frac{1}{2} \mathbf{V}_{1}^{T} Q \mathbf{V}_{1}\right) \exp \left(-\frac{1}{2} \mathbf{V}_{2}^{T} Q \mathbf{V}_{2}\right)
$$

The induced joint prior distribution of $\left(\begin{array}{l}\boldsymbol{\psi}_{1} \\ \boldsymbol{\psi}_{2}\end{array}\right)$, where $\boldsymbol{\psi}_{1}=\left(\psi_{11}, \psi_{12}, \ldots, \psi_{1} N_{s}\right)^{T}$ and $\boldsymbol{\psi}_{2}=\left(\psi_{21}, \psi_{22}, \ldots, \psi_{2 N_{s}}\right)^{T}$, is used for model fitting and can be represented as

$$
\left[\boldsymbol{\psi}_{1}, \boldsymbol{\psi}_{2} \mid A_{\psi}\right]=\left[\boldsymbol{\psi}_{1} \mid A_{\psi}\right]\left[\boldsymbol{\psi}_{2} \mid \boldsymbol{\psi}_{1}, A_{\psi}\right]
$$




$$
\propto \exp \left(-\frac{1}{2 a_{11}^{(\psi)^{2}}} \boldsymbol{\psi}_{1}^{T} Q \boldsymbol{\psi}_{1}\right) \exp \left(-\frac{1}{2 a_{22}^{(\psi)^{2}}}\left(\boldsymbol{\psi}_{2}-\frac{a_{12}^{(\psi)}}{a_{11}^{(\psi)}} \boldsymbol{\psi}_{1}\right)^{T} Q\left(\boldsymbol{\psi}_{2}-\frac{a_{12}^{(\psi)}}{a_{11}^{(\psi)}} \boldsymbol{\psi}_{1}\right)\right)
$$

For this section, let $\theta \mid \cdots$ indicate the full conditional distribution of $\theta$, where $\theta$ is an arbitrary parameter. For several quantities, we combine site-specific variables. For example, let $Y_{t}^{O}=\left(Y_{1 t}^{O}, \ldots, Y_{N_{s} t}^{O}\right)^{T}, Y_{t}^{P M}=\left(Y_{1 t}^{P M}, \ldots, Y_{N_{s} t}^{P M}\right)^{T}$, $\mathbf{X}_{t}=\operatorname{blockdiag}\left(\mathbf{x}_{i t}\right)$ and $\boldsymbol{\beta}_{k}=\left(\boldsymbol{\beta}_{k 1}, \ldots, \boldsymbol{\beta}_{k N_{s}}\right)^{T}$. In addition to previous terms, we also let $\mathbf{L}_{t}=$ blockdiag $\left(\mathbf{L}_{i t}\right)$ and $\gamma_{k}=\left(\gamma_{k 1}, \ldots, \gamma_{k N_{s}}\right)^{T}$. The full conditional distributions for this model are provided below.

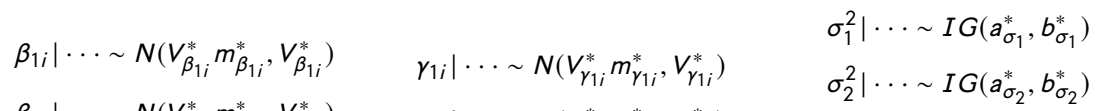

$$
\begin{aligned}
& \beta_{2 i}\left|\cdots \sim N\left(V_{\beta_{2 i}}^{*} m_{\beta_{2 i}}^{*}, V_{\beta_{2 i}}^{*}\right) \quad \gamma_{2 i}\right| \cdots \sim N\left(V_{\gamma_{2 i}}^{*} m_{\gamma_{2 i}}^{*}, V_{\gamma_{2 i}}^{*}\right) \quad \mathrm{V}_{1} \mid \cdots \sim N\left(V_{V_{1}}^{*} m_{V_{1}}^{*}, V_{V_{1}}^{*}\right) \\
& \beta_{01}\left|\cdots \sim N\left(V_{\beta_{01}}^{*} m_{\beta_{01}}^{*}, V_{\beta_{01}}^{*}\right) \quad \gamma_{01}\right| \cdots \sim N\left(V_{\gamma_{01}}^{*} m_{\gamma_{01}}^{*}, V_{\gamma_{01}}^{*}\right) \quad V_{2} \mid \cdots \sim N\left(V_{V_{2}}^{*} m_{V_{2}}^{*}, V_{V_{2}}^{*}\right) \\
& \beta_{02}\left|\cdots \sim N\left(V_{\beta_{02}}^{*} m_{\beta_{02}}^{*}, V_{\beta_{02}}^{*}\right) \quad \gamma_{02}\right| \cdots \sim N\left(V_{\gamma_{02}}^{*} m_{\gamma_{02}}^{*}, V_{\gamma_{02}}^{*}\right) \quad a_{11}^{(\psi)^{2}} \mid \cdots \sim I G\left(a_{a_{1}}^{*}, b_{a_{1}}^{*}\right) \text {, } \\
& \begin{array}{lll}
\Sigma_{\beta_{1}} \mid \cdots \sim I W\left(M_{\beta_{1}}^{*}, v_{\beta_{1}}^{*}\right) & \Sigma_{\gamma_{1}} \mid \cdots \sim I W\left(M_{\gamma_{1}}^{*}, v_{\gamma_{1}}^{*}\right) & a_{11} \mid \cdots \sim I G\left(a_{a_{1}}, b_{a_{1}}\right), \\
& \Sigma_{\gamma_{2}} \mid \cdots \sim I W\left(M_{\gamma_{2}}^{*}, v_{\gamma_{2}}^{*}\right) & a_{22}^{(\psi)^{2}} \mid \cdots \sim I G\left(a_{a_{2}}^{*}, b_{a_{2}}^{*}\right),
\end{array} \\
& \Sigma_{\beta_{2}}\left|\cdots \sim I W\left(M_{\beta_{2}}^{*}, v_{\beta_{2}}^{*}\right) \quad \Sigma_{\gamma_{2}}\right| \cdots \sim I W\left(M_{\gamma_{2}}^{*}, v_{\gamma_{2}}^{*}\right) \quad a_{12}^{(\psi)} \mid \cdots \sim N\left(V_{\psi}^{*} m_{\psi}^{*}, v_{\psi}^{*}\right)
\end{aligned}
$$

with

$$
\begin{aligned}
& a_{\sigma_{1}}^{*}=1+\frac{N_{s} \times N_{t}}{2} \\
& b_{\sigma_{1}}^{*}=1+\frac{1}{2} \sum_{t=1}^{N_{t}} \sum_{i=1}^{N_{s}}\left(Y_{i t}^{O}-\mathbf{x}_{i t}^{T} \boldsymbol{\beta}_{1 i}-\mathbf{L}_{i t}^{O T} \gamma_{1 i}-a_{11}^{(\psi)} V_{1 i}\right)^{2} \\
& a_{\sigma_{2}}^{*}=1+\frac{N_{s} \times N_{t}}{2} \\
& b_{\sigma_{2}}^{*}=1+\frac{1}{2} \sum_{t=1}^{N_{t}} \sum_{i=1}^{N_{s}}\left(Y_{i t}^{P M}-\mathbf{x}_{i t}^{T} \boldsymbol{\beta}_{2 i}-\mathbf{L}_{i t}^{P M^{T}} \boldsymbol{\gamma}_{2 i}-a_{12}^{(\psi)} V_{1 i}-a_{22}^{(\psi)} V_{2 i}\right)^{2} \\
& m_{\beta_{1 i}}^{*}=\Sigma_{\beta_{1}}^{-1} \beta_{01}+\frac{1}{\sigma_{1}^{2}} \sum_{t=1}^{N_{t}} \mathbf{x}_{i t}\left(Y_{i t}^{O}-\mathbf{L}_{i t}^{O T} \boldsymbol{\gamma}_{1 i}-a_{11}^{(\psi)} V_{1 i}\right) \\
& V_{\beta_{1 i}}^{*}=\left(\Sigma_{\beta_{1}}^{-1}+\frac{1}{\sigma_{1}^{2}} \sum_{t=1}^{N_{t}} \mathbf{x}_{i t} \mathbf{x}_{i t}^{T}\right)^{-1} \\
& m_{\beta_{2 i}}^{*}=\Sigma_{\beta_{2}}^{-1} \beta_{02}+\frac{1}{\sigma_{2}^{2}} \sum_{t=1}^{N_{t}} \mathbf{x}_{i t}\left(Y_{i t}^{P M}-\mathbf{L}_{i t}^{P M T} \gamma_{2 i}-a_{12}^{(\psi)} V_{1 i}-a_{22}^{(\psi)} V_{2 i}\right) \\
& V_{\beta_{2 i}}^{*}=\left(\Sigma_{\beta_{2}}^{-1}+\frac{1}{\sigma_{2}^{2}} \sum_{t=1}^{N_{t}} \mathbf{x}_{i t} \mathbf{x}_{i t}^{T}\right)^{-1} \\
& m_{\beta_{01}}^{*}=\sum_{i=1}^{N_{s}} \Sigma_{\beta_{1}}^{-1} \boldsymbol{\beta}_{1 i} \\
& V_{\beta_{01}}^{*}=\left(N_{s} \Sigma_{\beta_{1}}^{-1}+10^{-3} \mathbf{I}\right)^{-1} \\
& m_{\beta_{02}}^{*}=\sum_{i=1}^{N_{s}} \Sigma_{\beta_{2}}^{-1} \boldsymbol{\beta}_{2 i} \\
& V_{\beta_{02}}^{*}=\left(N_{s} \Sigma_{\beta_{2}}^{-1}+10^{-3} \mathbf{I}\right)^{-1} \\
& M_{\beta_{1}}^{*}=10^{3} \mathbf{I}+\sum_{i=1}^{N_{s}}\left(\boldsymbol{\beta}_{1 i}-\boldsymbol{\beta}_{01}\right)\left(\boldsymbol{\beta}_{1 i}-\boldsymbol{\beta}_{01}\right)^{T}
\end{aligned}
$$


White ET AL.

21

$$
\begin{aligned}
& v_{\beta_{1}}^{*}=N_{s}+p+1 \\
& \boldsymbol{M}_{\boldsymbol{\beta}_{2}}^{*}=10^{3} \mathbf{I}+\sum_{i=1}^{N_{s}}\left(\boldsymbol{\beta}_{2 i}-\boldsymbol{\beta}_{02}\right)\left(\boldsymbol{\beta}_{2 i}-\boldsymbol{\beta}_{02}\right)^{T} \\
& v_{\beta_{2}}^{*}=N_{s}+p+1 \\
& m_{\gamma_{1 i}}^{*}=\Sigma_{\gamma_{1}}^{-1} \gamma_{01}+\frac{1}{\sigma_{1}^{2}} \sum_{t=1}^{N_{t}} \mathbf{L}_{i t}^{O}\left(Y_{i t}^{O}-\mathbf{x}_{i t}^{T} \boldsymbol{\beta}_{1 i}-a_{11}^{(\psi)} V_{1 i}\right) \\
& V_{\gamma_{1 i}}^{*}=\left(\Sigma_{\gamma_{1}}^{-1}+\frac{1}{\sigma_{1}^{2}} \sum_{t=1}^{N_{t}} \mathbf{L}_{i t}^{O} \mathbf{L}_{i t}^{O^{T}}\right)^{-1} \\
& m_{\gamma_{2 i}}^{*}=\Sigma_{\gamma_{2}}^{-1} \gamma_{02}+\frac{1}{\sigma_{2}^{2}} \sum_{t=1}^{N_{t}} \mathbf{L}_{i t}^{P M}\left(Y_{i t}^{P M}-\mathbf{x}_{i t}^{T} \boldsymbol{\beta}_{2 i}-a_{12}^{(\psi)} V_{1 i}-a_{22}^{(\psi)} V_{2 i}\right) \\
& \Sigma_{\gamma_{2 i}}^{*}=\left(\Sigma_{\gamma_{2}}^{-1}+\frac{1}{\sigma_{2}^{2}} \sum_{t=1}^{N_{t}} \mathbf{L}_{i t}^{P M} \mathbf{L}_{i t}^{P M^{T}}\right)^{-1} \\
& m_{\gamma_{01}}^{*}=\sum_{i=1}^{N_{s}} \Sigma_{\gamma_{1}}^{-1} \gamma_{1 i} \\
& V_{\gamma_{01}}^{*}=\left(N_{s} \Sigma_{\gamma_{1}}^{-1}+10^{-3} \mathrm{I}\right)^{-1} \\
& m_{\gamma_{02}}^{*}=\sum_{i=1}^{N_{s}} \Sigma_{\gamma_{2}}^{-1} \gamma_{2 i} \\
& V_{\gamma_{02}}^{*}=\left(N_{s} \Sigma_{\gamma_{2}}^{-1}+10^{-3} I\right)^{-1} \\
& M_{\gamma_{1}}^{*}=10^{3} \mathbf{I}+\sum_{i=1}^{N_{s}}\left(\gamma_{1 i}-\gamma_{01}\right)\left(\gamma_{1 i}-\gamma_{01}\right)^{T} \\
& v_{\gamma_{1}}^{*}=N_{s}+n_{1 /}+1 \\
& M_{\gamma_{2}}^{*}=10^{3} \mathbf{I}+\sum_{i=1}^{N_{s}}\left(\gamma_{2 i}-\gamma_{02}\right)\left(\gamma_{2 i}-\gamma_{02}\right)^{T} \\
& v_{\gamma_{2}}^{*}=N_{s}+n_{2 l}+1 \\
& m_{V_{1}}^{*}=\frac{a_{11}^{(\psi)}}{\sigma_{1}^{2}} \sum_{i=1}^{N_{t}}\left(Y_{t}^{O}-\mathbf{X}_{t} \boldsymbol{\beta}_{1}-\mathbf{L}_{t}^{O} \boldsymbol{\gamma}_{1}\right)+ \\
& \frac{a_{12}^{(\psi)}}{\sigma_{2}^{2}} \sum_{i=1}^{N_{t}}\left(Y_{t}^{P M}-\mathbf{X}_{t} \boldsymbol{\beta}_{2}-\mathbf{L}_{t}^{P M} \gamma_{2}-a_{22}^{(\psi)} \mathbf{V}_{2}\right) \\
& V_{V_{1}}^{*}=\left(Q_{1}+\left[\frac{a_{11}^{(\psi)^{2}} N_{t}}{\sigma_{1}^{2}}+\frac{a_{12}^{(\psi)^{2}} N_{t}}{\sigma_{2}^{2}}\right] \mathrm{I}\right)^{-1} \\
& m_{V_{2}}^{*}=\frac{a_{22}^{(\psi)}}{\sigma_{2}^{2}} \sum_{i=1}^{N_{t}}\left(Y_{t}^{P M}-\mathbf{X}_{t} \boldsymbol{\beta}_{2}-\mathbf{L}_{t}^{P M} \gamma_{2}-a_{12}^{(\psi)} \mathbf{V}_{1}\right) \\
& V_{V_{2}}^{*}=\left(Q_{2}+\mathbf{I} \frac{a_{22}^{(\psi)^{2}} N_{t}}{\sigma_{2}^{2}}\right)^{-1} \\
& a_{a_{1}}^{*}=1+N_{s} / 2 \\
& b_{a_{1}}^{*}=1+\frac{1}{2} \boldsymbol{\psi}_{1}^{T} Q_{1} \boldsymbol{\psi}_{1} \\
& a_{a_{2}}^{*}=1+N_{s} / 2 \\
& b_{a_{2}}^{*}=1+\frac{1}{2}\left(\boldsymbol{\psi}_{2}-\frac{a_{12}^{(\psi)}}{a_{11}^{(\psi)}} \boldsymbol{\psi}_{1}\right)^{T} Q\left(\boldsymbol{\psi}_{2}-\frac{a_{12}^{(\psi)}}{a_{11}^{(\psi)}} \boldsymbol{\psi}_{1}\right) \\
& m_{\psi}^{*}=\frac{1}{\sigma_{2}^{2}} \sum_{t=1}^{N_{t}} \sum_{i=1}^{N_{s}} V_{1 i}\left(Y_{i t}^{P M}-\mathbf{L}_{i t}^{P M^{T}} \gamma_{2 i}-\mathbf{x}_{i t}^{T} \beta_{2 i}-a_{22}^{(\psi)} V_{2 i}\right)
\end{aligned}
$$




$$
V_{\psi}^{*}=\left(10^{-3}+\frac{1}{\sigma_{2}^{2}} \sum_{t=1}^{N_{t}} \sum_{i=1}^{N_{s}} V_{1 i}^{2}\right)^{-1}
$$

\section{B | FULL CONDITIONAL DISTRIBUTIONS FOR HETEROSCEDASTIC AR MODEL}

We rely on some of the details presented in Appendix A for the CAR terms. We give the full conditional distributions for the heteroscedastic model specified in Section 3 where the variance is a function of the hour of the day $h(t)$. For this section, let $\theta \mid \ldots$ indicate the full conditional distribution of $\theta$, where $\theta$ is an arbitrary parameter. We again combine several quantities for site-specific variables. Let $Y_{t}^{O}=\left(Y_{1 t}^{O}, \ldots, Y_{N_{s} t}^{O}\right)^{T}, Y_{t}^{P M}=\left(Y_{1 t}^{P M}, \ldots, Y_{N_{s} t}^{P M}\right)^{T}, \mathbf{X}_{t}=\operatorname{blockdiag}\left(\mathbf{x}_{i t}\right)$ and $\boldsymbol{\beta}_{k}=\left(\boldsymbol{\beta}_{k 1}, \ldots, \boldsymbol{\beta}_{k N_{s}}\right)^{T}$. In addition to previous terms, we also let $\mathbf{L}_{t}=$ blockdiag $\left(\mathbf{L}_{i t}\right)$ and $\boldsymbol{\gamma}_{k}=\left(\gamma_{k 1}, \ldots, \gamma_{k N_{s}}\right)^{T}$. The full conditional distributions for this model are provided below. If posterior parameters are not given below, then they are identical to those given for the homoscedastic model in Appendix A.

$$
\begin{aligned}
& \beta_{1 i}\left|\cdots \sim N\left(V_{\beta_{1 i}}^{*} m_{\beta_{1 i}}^{*}, V_{\beta_{1 i}}^{*}\right) \quad \gamma_{1 i}\right| \cdots \sim N\left(V_{\gamma_{1 i}}^{*} m_{\gamma_{1 i}}^{*}, V_{\gamma_{1 i}}^{*}\right) \\
& \beta_{2 i}\left|\cdots \sim N\left(V_{\beta_{2 i}}^{*} m_{\beta_{2 i}}^{*}, V_{\beta_{2 i}}^{*}\right) \quad \gamma_{2 i}\right| \cdots \sim N\left(V_{\gamma_{2 i}}^{*} m_{\gamma_{2 i}}^{*}, V_{\gamma_{2 i}}^{*}\right) \\
& \beta_{01}\left|\cdots \sim N\left(V_{\beta_{01}}^{*} m_{\beta_{01}}^{*}, V_{\beta_{01}}^{*}\right) \quad \gamma_{01}\right| \cdots \sim N\left(V_{\gamma_{01}}^{*} m_{\gamma_{01}}^{*}, V_{\gamma_{01}}^{*}\right) \\
& \beta_{02}\left|\cdots \sim N\left(V_{\beta_{02}}^{*} m_{\beta_{02}}^{*}, V_{\beta_{02}}^{*}\right) \quad \gamma_{02}\right| \cdots \sim N\left(V_{\gamma_{02}}^{*} m_{\gamma_{02}}^{*}, V_{\gamma_{02}}^{*}\right) \\
& \Sigma_{\beta_{1}}\left|\cdots \sim \operatorname{IW}\left(M_{\beta_{1}}^{*}, v_{\beta_{1}}^{*}\right) \quad \Sigma_{\gamma_{1}}\right| \cdots \sim \operatorname{IW}\left(M_{\gamma_{1}}^{*}, v_{\gamma_{1}}^{*}\right) \\
& \Sigma_{\beta_{2}}\left|\cdots \sim I W\left(M_{\beta_{2}}^{*}, v_{\beta_{2}}^{*}\right) \quad \Sigma_{\gamma_{2}}\right| \cdots \sim I W\left(M_{\gamma_{2}}^{*}, v_{\gamma_{2}}^{*}\right) \\
& \sigma_{1 q}^{2} \mid \cdots \sim I G\left(a_{\sigma_{1 q}}^{*}, b_{\sigma_{1 q}}^{*}\right) \\
& \sigma_{2 q}^{2} \mid \cdots \sim I G\left(a_{\sigma_{2 q}}^{*}, b_{\sigma_{2 q}}^{*}\right) \\
& \mathrm{V}_{1} \mid \cdots \sim N\left(V_{V_{1}}^{*} m_{V_{1}}^{*}, V_{V_{1}}^{*}\right) \\
& \mathbf{V}_{2} \mid \cdots \sim N\left(V_{V_{2}}^{*} m_{V_{2}}^{*}, V_{V_{2}}^{*}\right) \\
& a_{11}^{(\psi)^{2}} \mid \cdots \sim I G\left(a_{a_{1}}^{*}, b_{a_{1}}^{*}\right), \\
& a_{22}^{(\psi)^{2}} \mid \cdots \sim I G\left(a_{a_{2}}^{*}, b_{a_{2}}^{*}\right), \\
& a_{12}^{(\psi)} \mid \cdots \sim N\left(V_{\psi}^{*} m_{\psi}^{*}, V_{\psi}^{*}\right)
\end{aligned}
$$

with

$$
\begin{aligned}
& a_{\sigma_{1 q}}^{*}=1+\frac{N_{s} \times N_{t}}{2 N_{h}} \\
& b_{\sigma_{1 q}}^{*}=1+\frac{1}{2} \sum_{t=1}^{N_{t}} \sum_{i=1}^{N_{s}}\left(Y_{i t}^{O}-\mathbf{x}_{i t}^{T} \boldsymbol{\beta}_{1 i}-\mathbf{L}_{i t}^{O^{T}} \boldsymbol{\gamma}_{1 i}-a_{11}^{(\psi)} V_{1 i}\right)^{2} \mathbf{1}(h(t)=q) \\
& a_{\sigma_{2 q}}^{*}=1+\frac{N_{s} \times N_{t}}{2 N_{h}} \\
& b_{\sigma_{2 q}}^{*}=1+\frac{1}{2} \sum_{t=1}^{N_{t}} \sum_{i=1}^{N_{s}}\left(Y_{i t}^{P M}-\mathbf{x}_{i t}^{T} \boldsymbol{\beta}_{2 i}-\mathbf{L}_{i t}^{P M^{T}} \gamma_{2 i}-a_{12}^{(\psi)} V_{1 i}-a_{22}^{(\psi)} V_{2 i}\right)^{2} \mathbf{1}(h(t)=q) \\
& m_{\beta_{1 i}}^{*}=\Sigma_{\beta_{1}}^{-1} \beta_{01}+\sum_{t=1}^{N_{t}} \frac{1}{\sigma_{1 h(t)}^{2}} \mathbf{x}_{i t}\left(Y_{i t}^{O}-\mathbf{L}_{i t}^{O T} \boldsymbol{\gamma}_{1 i}-a_{11}^{(\psi)} V_{1 i}\right) \\
& V_{\beta_{1 i}}^{*}=\left(\Sigma_{\beta_{1}}^{-1}+\sum_{t=1}^{N_{t}} \frac{1}{\sigma_{1 h(t)}^{2}} \mathbf{x}_{i t} \mathbf{x}_{i t}^{T}\right)^{-1} \\
& m_{\beta_{2 i}}^{*}=\Sigma_{\beta_{2}}^{-1} \beta_{02}+\sum_{t=1}^{N_{t}} \frac{1}{\sigma_{2 h(t)}^{2}} \mathbf{x}_{i t}\left(Y_{i t}^{P M}-\mathbf{L}_{i t}^{P M T} \boldsymbol{\gamma}_{2 i}-a_{12}^{(\psi)} V_{1 i}-a_{22}^{(\psi)} V_{2 i}\right) \\
& V_{\beta_{2 i}}^{*}=\left(\Sigma_{\beta_{2}}^{-1}+\sum_{t=1}^{N_{t}} \frac{1}{\sigma_{2 h(t)}^{2}} \mathbf{x}_{i t} \mathbf{x}_{i t}^{T}\right)^{-1} \\
& m_{\gamma_{1 i}}^{*}=\Sigma_{\gamma_{1}}^{-1} \gamma_{01}+\sum_{t=1}^{N_{t}} \frac{1}{\sigma_{1 h(t)}^{2}} \mathbf{L}_{i t}^{O}\left(Y_{i t}^{O}-\mathbf{x}_{i t}^{T} \boldsymbol{\beta}_{1 i}-a_{11}^{(\psi)} V_{1 i}\right)
\end{aligned}
$$




$$
\begin{aligned}
& V_{\gamma_{1 i}}^{*}=\left(\Sigma_{\gamma_{1}}^{-1}+\sum_{t=1}^{N_{t}} \frac{1}{\sigma_{1 h(t)}^{2}} \mathbf{L}_{i t}^{O} \mathbf{L}_{i t}^{O T}\right)^{-1} \\
& m_{\gamma_{2 i}}^{*}=\Sigma_{\gamma_{2}}^{-1} \gamma_{02}+\sum_{t=1}^{N_{t}} \frac{1}{\sigma_{2 h(t)}^{2}} \mathbf{L}_{i t}^{P M}\left(Y_{i t}^{P M}-\mathbf{x}_{i t}^{T} \boldsymbol{\beta}_{2 i}-a_{12}^{(\psi)} V_{1 i}-a_{22}^{(\psi)} V_{2 i}\right) \\
& V_{\gamma_{2 i}}^{*}=\left(\Sigma_{\gamma_{2}}^{-1}+\sum_{t=1}^{N_{t}} \frac{1}{\sigma_{2 h(t)}^{2}} \mathbf{L}_{i t}^{P M} \mathbf{L}_{i t}^{P M^{T}}\right)^{-1} \\
& m_{V_{1}}^{*}=a_{11}^{(\psi)} \sum_{t=1}^{N_{t}} \frac{1}{\sigma_{1 h(t)}^{2}}\left(Y_{t}^{O}-\mathbf{X}_{t} \boldsymbol{\beta}_{1}-\mathbf{L}_{t}^{O} \boldsymbol{\gamma}_{1}\right)+ \\
& a_{12}^{(\psi)} \sum_{t=1}^{N_{t}} \frac{1}{\sigma_{2 h(t)}^{2}}\left(Y_{t}^{P M}-\mathbf{X}_{t} \boldsymbol{\beta}_{2}-\mathbf{L}_{t}^{P M} \gamma_{2}-a_{22}^{(\psi)} \mathbf{V}_{2}\right) \\
& V_{V_{1}}^{*}=\left(Q_{1}+\left[a_{11}^{(\psi)^{2}} \frac{N_{t}}{N_{h}} \sum_{q=1}^{24} \sigma_{1 q}^{2}+a_{12}^{(\psi)^{2}} \frac{N_{t}}{N_{h}} \sum_{q=1}^{24} \sigma_{2 q}^{2}\right] 1\right)^{-1} \\
& m_{V_{2}}^{*}=\frac{a_{22}^{(\psi)}}{\sigma_{2}^{2}} \sum_{i=1}^{N_{t}}\left(Y_{t}^{P M}-\mathbf{X}_{t} \boldsymbol{\beta}_{2}-\mathbf{L}_{t}^{P M} \gamma_{2}-a_{12}^{(\psi)} \mathbf{V}_{1}\right) \\
& V_{V_{2}}^{*}=\left(Q_{2}+\mathbf{I}_{22}^{(\psi)^{2}} \frac{N_{t}}{N_{h}} \sum_{q=1}^{24} \sigma_{2 q}^{2}\right)^{-1} \\
& m_{\psi}^{*}=\sum_{t=1}^{N_{t}} \frac{1}{\sigma_{2 h(t)}^{2}} \sum_{i=1}^{N_{s}} V_{1 i}\left(Y_{i t}^{P M}-\mathbf{L}_{i t}^{P M^{T}} \gamma_{2 i}-\mathbf{x}_{i t}^{T} \beta_{2 i}-a_{22}^{(\psi)} V_{2 i}\right) \\
& V_{\psi}^{*}=\left(10^{-3}+\sum_{t=1}^{N_{t}} \frac{1}{\sigma_{2 h(t)}^{2}} \sum_{i=1}^{N_{s}} V_{1 i}^{2}\right)^{-1}
\end{aligned}
$$

\section{C $\mid$ PREDICTION OF HELD-OUT DATA}

For model validation, we hold out $10 \%$ of the data and impute or update these held-out values each step of the Gibbs sampler which is described below.

$$
\begin{aligned}
& \mu_{1 i t}=\mathbf{x}_{i(t-1)}^{T} \boldsymbol{\beta}_{1 i}+\mathbf{L}_{i t}^{O^{T}} \gamma_{1 i}+\psi_{1 i}, \\
& \mu_{2 i t}=\mathbf{x}_{i(t-1)}^{T} \boldsymbol{\beta}_{2 i}+\mathbf{L}_{i t}^{P M^{T}} \gamma_{2 i}+\psi_{2 i},
\end{aligned}
$$

and let $Y_{i t}^{O} \mid \cdots$ and $Y_{i t}^{P M} \mid \cdots$ denote the full conditional distributions of missing observations. For the heteroscedastic model, the full conditional distributions for the missing data are

$$
\begin{array}{r}
Y_{i t}^{O} \mid \cdots \sim N\left(\tau_{1 i t}^{*} \mu_{1 i t}^{*}, \tau_{1 i t}^{*}\right) \\
Y_{i t}^{P M} \mid \cdots \sim N\left(\tau_{2 i t}^{*} \mu_{2 i t}^{*}, \tau_{2 i t}^{*}\right)
\end{array}
$$

with

$$
\begin{aligned}
& \tau_{1 i t}^{*}=\left(\frac{1}{\sigma_{1 t}^{2}}+\sum_{j=1}^{n_{1 /}} \frac{\gamma_{1 j}^{2}}{\sigma_{1\left(t+l_{1 j}\right)}^{2}}\right)^{-1} \\
& \mu_{1 i t}^{*}=\mu_{1 i t}+\sum_{j=1}^{n_{1 /}} \frac{\gamma_{1 i j}\left(Y_{i\left(t+l_{1 j}\right)}^{O}-m_{1 i\left(t+l_{1 j}\right)}+\gamma_{1 i j} Y_{i t}^{O}\right)}{\sigma_{1\left(t+l_{1 j}\right)}^{2}}
\end{aligned}
$$




$$
\begin{aligned}
& \tau_{2 i t}^{*}=\left(\frac{1}{\sigma_{2 t}^{2}}+\sum_{j=1}^{n_{2 l}} \frac{\gamma_{2 j}^{2}}{\sigma_{2\left(t+l_{2 j}\right)}^{2}}\right)^{-1} \\
& \mu_{2 i t}^{*}=\mu_{2 i t}+\sum_{j=1}^{n_{2 l}} \frac{\gamma_{2 i j}\left(Y_{i\left(t+l_{2 j}\right)}^{P M}-m_{2 i\left(t+l_{2 j}\right)}+\gamma_{2 i j} Y_{i t}^{P M}\right)}{\sigma_{2\left(t+l_{2 j}\right)}^{2}},
\end{aligned}
$$

where $I_{1 j}$ is the $j^{\text {th }}$ lag for ozone with coefficient $\gamma_{1 i j}$ and $l_{2 j}$ is the $j^{\text {th }}$ lag for $\mathrm{PM}_{10}$ with coefficient $\gamma_{1 i j}$. The imputation method for the homoscedastic model is a special case of the heteroscedastic model. 